\title{
The fundamental role of symmetry in nuclear models
}

\author{
Lecture notes for the International Scientific Meeting on Nuclear Physics \\ at La Rábida, Huelva (Spain), September 9-13, 2012.
}

D. J. Rowe

Department of Physics, University of Toronto, Toronto, Ontario M5S 1A7, Canada

\begin{abstract}
The purpose of these lectures is to illustrate how symmetry and pattern recognition play essential roles in the progression from experimental observation to an understanding of nuclear phenomena in terms of interacting neutrons and protons. We do not discuss weak interactions nor relativistic and sub-nucleon degrees of freedom. The explicit use of symmetry and the power of algebraic methods, in combination with analytical and geometrical methods are illustrated by their use in deriving a shell-model description of nuclear rotational dynamics and the structure of deformed nuclei.
\end{abstract}

Keywords: Symmetry, dynamical group, spectrum generating algebra, nuclear collective model, microscopic structure of deformed nuclei, the symplectic shell model.

PACS: 21.60.-n, 21.60.Ev, 21.60.Fw, 02.20.-a

\section{INTRODUCTION}

It has become common practice in nuclear physics to make a distinction between algebraic and geometric models. This is convenient for some purposes but, in general, it is misleading because almost all models in quantum mechanics have expressions in algebraic terms. In fact, many-particle quantum mechanics is fundamentally a unitary representation of the Lie algebra of one-body operators and its observables are polynomials in the elements of this Lie algebra.

In proceeding towards a microscopic understanding of some nuclear phenomena, it is profitable to follow a sequence of steps along the following lines:

(i) Observe the phenomena in many situations until its pattern becomes evident.

(ii) Develop a phenomenological model to explain the observations and suggest new observations to ascertain the consistency of the model.

(iii) Repeat steps (i) and (ii) to refine the model, assess its reliability and domain of validity, and identify its algebraic structure.

(iv) If a model provides a successful understanding of the phenomena, the next step is to understand the model in terms of interacting nucleons. This is achieved if one is able to express the observables of the model in terms of nucleon coordinates and momenta. In the process, it is likely that the model will only be realisable in some limit or as an approximation to a more complex microscopic theory. It will then be of fundamental importance to determine if the limitations are consistent with the 


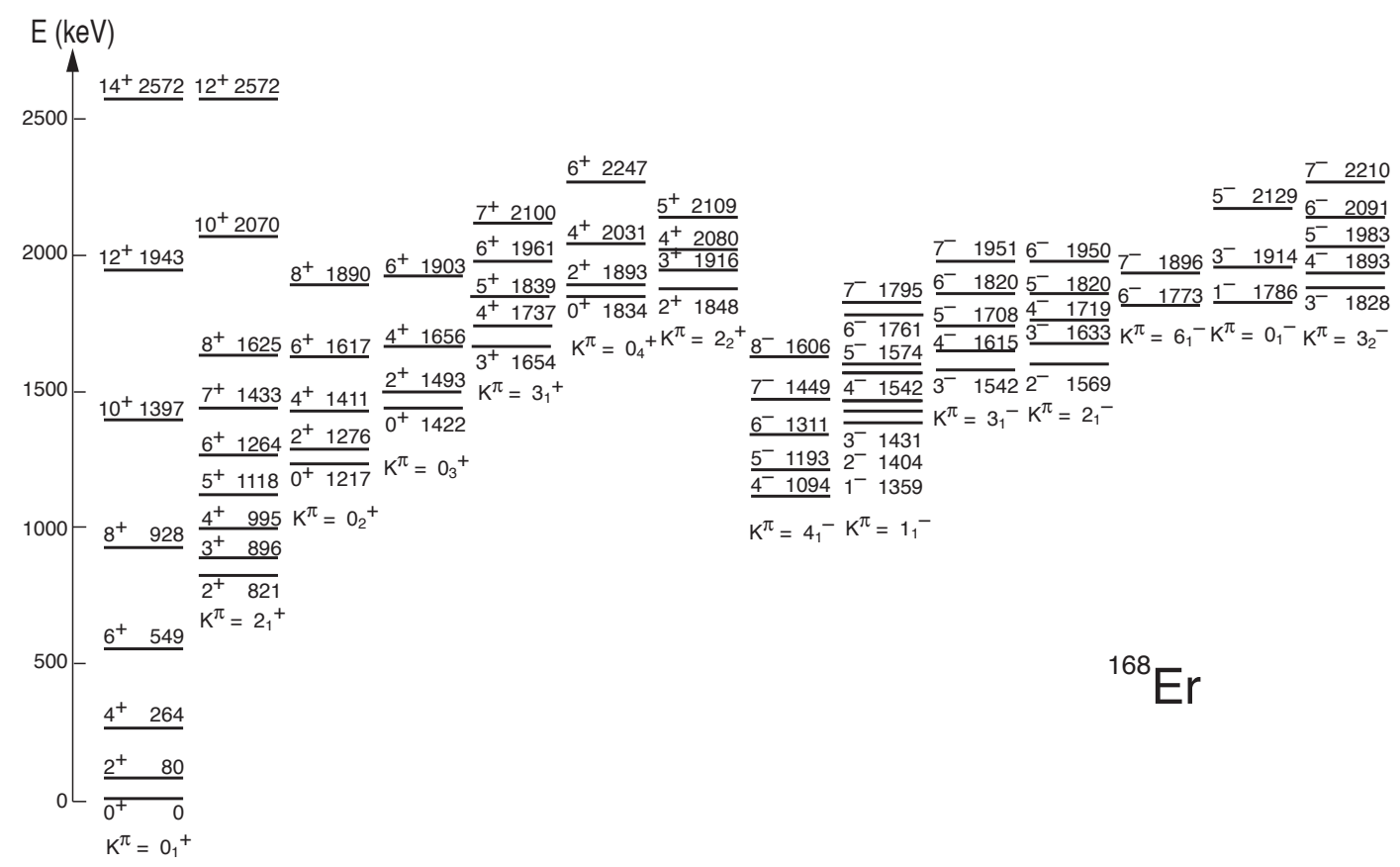

FIGURE 1. The low-lying states of ${ }^{168} \mathrm{Er}$ arranged into rotational bands.

experimental observations. As the following examples will illustrate, this exercise can also lead to improved phenomenological models with clearer microscopic foundations.

(v) Having found a microscopically successful model, whose representations are capable of explaining a range of physical observations, the next goal is to make use of its algebraic structure to identify an associated shell-model coupling scheme that will be appropriate for a shell-model description of the phenomena with realistic nucleon-nucleon interactions.

The remarkable fact is that by such sequences of steps, one has obtained shell model coupling schemes appropriate for some of the dominant characteristics of nuclei. For example, the basic harmonic-oscillator shell-model, proposed to explain the strong binding energy of so-called magic nuclei in terms of closed-shell structures, has provided the independent-particle basis for the standard shell model given by nucleons in a meanfield with spin-orbit interactions. Pairing models of singly closed-shell nuclei lead to the standard $j j$-coupling scheme of Flowers [1]; and nuclear rotational models lead to the Elliott SU(3) coupling scheme [2] for light nuclei.

In the following we start from the observation of rotational bands organised as shown, for example, in Fig. 1 and the interpretations of such bands in the Bohr model and proceed to their implications for a shell-model theory of strongly deformed nuclei. However, before embarking on this process, we first recall some of the primary symmetry concepts that will be used. 


\section{SYMMETRY GROUPS, DYNAMICAL GROUPS AND SPECTRUM GENERATING ALGEBRAS}

Definition: A symmetry group of a system is a group of transformations of the system that leave its Hamiltonian invariant.

There are several variations in the literature on the definition of a dynamical group [3]. The following definition is most useful for present purposes.

Definition: A dynamical group for a Hamiltonian $\hat{H}$ is a Lie group of unitary transformations of the Hilbert space $\mathbb{H}$ of a system with Hamiltonian $\hat{H}$ such that the subspaces of $\mathbb{H}$ that are invariant under the transformations of the dynamical group are spanned by eigenstates of $\hat{H}$.

Consider, for example, a particle in ordinary three space moving in a central force potential $V(r)$ such that the Hamiltonian is $\mathrm{SO}(3)$ invariant. Basis wave functions for the particle's Hilbert space are given by products of radial wave functions and spherical harmonics

$$
\psi_{n l m}(r, \theta, \varphi)=R_{n l}(r) Y_{l m}(\theta \varphi),
$$

where $l$ and $m$ are angular-momentum quantum numbers and $n$ is a radial quantum number. The Hamiltonian matrix in this basis is given by

$$
H_{n m, n^{\prime} m^{\prime}}^{l}=\left\langle\psi_{n l m}|\hat{H}| \psi_{n^{\prime} l m^{\prime}}\right\rangle
$$

which, because $\mathrm{SO}(3)$ is a symmetry group, has $l$ as a good quantum number. In addition, because $\hat{H}$ is $\mathrm{SO}(3)$ invariant, the Hamiltonian matrix elements are diagonal in $m$ and independent of its value, i.e.,

$$
H_{n m, n^{\prime} m^{\prime}}^{l}=\delta_{m, m^{\prime}} H_{n, n^{\prime}}^{l}
$$

and $m$ is also a good quantum number. Thus, to obtain the energy spectrum for the particle, it remains only to diagonalise each $H^{l}$ with respect to the radial quantum number $n$ to obtain its eigenfunctions

$$
\Psi_{\alpha l m}=\sum_{n} C_{\alpha n} \psi_{n l m}
$$

This process is greatly simplified by the fact that the central force problem has a dynamical group $\mathrm{SU}(1,1)$ for which the set of states $\left\{\psi_{n l m} ; n=0,1,2, \ldots\right\}$ for each pair of $l m$ values carries an irreducible representation.

Definition: A spectrum generating algebra (SGA) for a Hamiltonian is a Lie algebra such that the Hamiltonian can be expressed (most usefully as polynomials) in terms of its elements.

An SGA $\mathfrak{g}$ is most useful because its representation theory enables one to calculate the matrix elements of any $X \in \mathfrak{g}$ and hence the matrix elements of the Hamiltonian. A 
Hamiltonian may have many SGA's. A particularly useful choice is one for which the important observables of the system have simple expressions in terms of its Lie algebra $\mathfrak{g}$, e.g., as linear or quadratic polynomials of Lie algebra elements.

An SGA for the above central-force problem is the Lie algebra su(1,1) whose infinitesimal generators include the square of the radial coordinate, $r^{2}$, of the particle and the square of it momentum, $p^{2}$.

A system may have many symmetry groups and many dynamical groups. For example, the group generated by an SGA for a Hamiltonian is a dynamical group for that Hamiltonian.

\section{THE BOHR COLLECTIVE MODEL}

The Bohr model is a liquid-drop model with quadrupole shape coordinates defined for its surface radius by

$$
R(\theta, \varphi)=R_{0}\left[1+\sum_{v} \alpha_{v} Y_{2 v}^{*}(\theta, \varphi)+\ldots\right]
$$

It is quantised by the introduction of quadrupole shape observables $\left\{\hat{\alpha}_{v}\right\}$ and canonical momenta $\left\{\hat{\pi}^{v}\right\}$ that satisfy the Heisenberg commutation relations

$$
\left[\hat{\alpha}_{\mu}, \hat{\pi}^{v}\right]=\mathrm{i} \hbar \delta_{\mu}^{v}
$$

and act as operators on a Hilbert space $\mathbb{H}$ of square-integrable wave functions of the shape variables, according to the equations

$$
\hat{\alpha}_{v} \psi(\alpha)=\alpha_{v} \psi(\alpha), \quad \hat{\pi}^{v}=-\mathrm{i} \hbar \frac{\partial}{\partial \alpha_{v}} \psi(\alpha), \quad v=0, \pm 1, \pm 2 .
$$

A Hamiltonian for the model is then an $\mathrm{SO}(3)$-invariant operator of the form

$$
\hat{H}=\frac{1}{B} \hat{\pi} \cdot \hat{\pi}+V(\alpha),
$$

where $V(\alpha)$ is a rotationally-invariant potential energy (a function of the coordinates) and $B$ is a mass parameter.

The model has geometric and algebraic structures that make it easy to use, but which have only recently been exploited in what is known as the Algebraic Collective Model $(\mathrm{ACM})[4,5,6,7]$. The coordinate space of this model is the real five-dimensional Euclidean space $\mathbb{R}^{5}$. This space has much in common with the more familiar threedimensional space $\mathbb{R}^{3}$ and similarly, for a rotationally-invariant Hamiltonian, it is most naturally assigned spherical polar coordinates. Apart from the origin (which is a point of measure zero), the Euclidean space $\mathbb{R}^{3}$ is a tensor product of a radial line and a twosphere of unit radius as illustrated in Fig. 2(a). Similarly, to within a point of measure zero, the Euclidean space $\mathbb{R}^{5}$ is a tensor product of a radial line and a four-sphere of unit radius. Thus, whereas $\mathbb{R}^{3}$ is assigned spherical polar coordinates $(r, \theta, \varphi)$, where $r$ is a radial coordinate and $(\theta, \varphi)$ are coordinates for a point on the two-sphere, the 


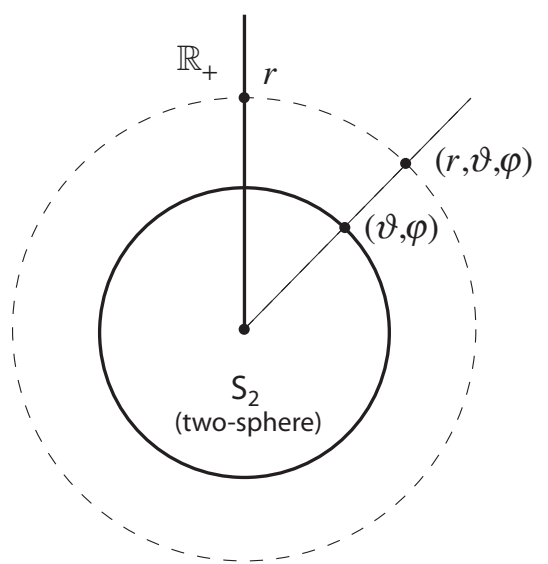

(a) $\mathbb{R}^{3} \sim \mathbb{R}_{+} \times S_{2}$

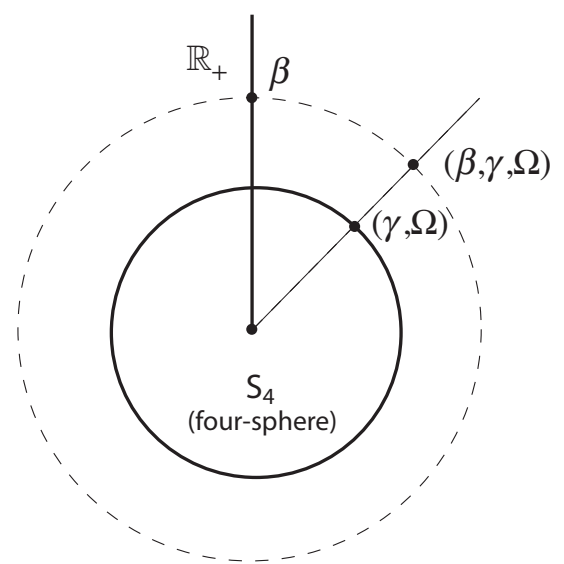

(b) $\mathbb{R}{ }^{5} \mathbb{R}_{+} \times S_{4}$

FIGURE 2. Spherical polar coordinates (a) for a three-dimensional and (b) for a five-dimensional Euclidean space.

space $\mathbb{R}^{5}$ is assigned spherical polar coordinates $(\beta, \gamma, \Omega)$, where now $\beta$ is a radial coordinate, defined by $\beta^{2}=\alpha \cdot \alpha$, which together with an angle coordinate $\gamma$ determine the triaxial shape of a rotor; $\Omega$ denotes the orientation angles of this rotor. Together, the angle coordinates $(\gamma, \Omega)$ complete a system of spherical coordinates for the four-sphere. Thus, whereas the Hilbert space for $\mathbb{R}^{3}$ is the tensor product

$$
\mathscr{L}^{2}\left(\mathbb{R}^{3}\right)=\mathscr{L}^{2}\left(\mathbb{R}_{+}\right) \times \mathscr{L}^{2}\left(S_{2}\right)
$$

and is spanned by functions $\left\{\psi_{n l m}(r, \theta, \varphi)=R_{l n}(r) Y_{l m}(\theta, \varphi)\right\}$, where $\left\{R_{l n}(r)\right\}$ is a basis for a unitary irrep (irreducible representation) of the group $\mathrm{SU}(1,1)$ and $\left\{Y_{l m}(\theta, \varphi)\right\}$ is a basis of spherical harmonics for the two-sphere, the Hilbert space for $\mathbb{R}^{5}$ is the tensor product

$$
\mathscr{L}^{2}\left(\mathbb{R}^{5}\right)=\mathscr{L}^{2}\left(\mathbb{R}_{+}\right) \times \mathscr{L}^{2}\left(S_{4}\right)
$$

and is spanned by functions $\left\{\mathscr{R}_{v n}(\beta) \mathscr{Y}_{v \alpha L M}(\gamma, \Omega)\right\}$, where $\left\{\mathscr{R}_{v n}(\beta)\right\}$ is again a basis for a unitary irrep of the group $\mathrm{SU}(1,1)$ and $\left\{\mathscr{Y}_{v \alpha L M}(\gamma, \Omega)\right\}$ is a basis of $\mathrm{SO}(5)$ spherical harmonics for the four-sphere.

Matrix elements of operators of interest on the Hilbert space $\mathscr{L}^{2}\left(\mathbb{R}^{3}\right)$ can be calculated algebraically. This is because explicit expressions are known [8] for the matrix elements of $r, 1 / r, r^{2}, 1 / r^{2}, \nabla^{2}$, for $\mathrm{SO}(3)$ spherical harmonics, for Clebsch-Gordan coefficients, and for the $\mathrm{SO}(3)$-reduced matrix elements

$$
\left\langle Y_{l_{3}}\left\|\hat{Y}_{l_{2}}\right\| Y_{l_{1}}\right\rangle=\sqrt{\frac{\left(2 l_{2}+1\right)\left(2 l_{1}+1\right)}{4 \pi}}\left(l_{1} 0 l_{2} 0 \mid l_{3} 0\right) .
$$

Similar expressions are now known for the Hilbert space $\mathscr{L}^{2}\left(\mathbb{R}^{5}\right)$ of the Bohr model. By simple algebraic methods [8], we obtain explicit expressions for the matrix elements of $\beta, 1 / \beta, \beta^{2}, 1 / \beta^{2}$ and $\nabla^{2}$. From the basic $\mathrm{SO}(5)$ spherical harmonics

$$
\mathscr{Y}_{1122}(\gamma, \Omega) \propto \cos \gamma \mathscr{D}_{02}^{2}+\sqrt{\frac{1}{2}} \sin \gamma\left(\mathscr{D}_{22}^{2}(\Omega)+\mathscr{D}_{-2,2}^{2}(\Omega)\right),
$$




$$
\begin{aligned}
& \mathscr{Y}_{2122}(\gamma, \Omega) \propto \cos 2 \gamma \mathscr{D}_{02}^{2}-\sqrt{\frac{1}{2}} \sin 2 \gamma\left(\mathscr{D}_{22}^{2}(\Omega)+\mathscr{D}_{-2,2}^{2}(\Omega)\right), \\
& \mathscr{Y}_{3100}(\gamma, \Omega) \propto \cos 3 \gamma, \\
& \mathscr{Y}_{3133}(\gamma, \Omega) \propto \sqrt{\frac{1}{2}} \sin 3 \gamma\left(\mathscr{D}_{23}^{3}(\Omega)-\mathscr{D}_{-2,3}^{2}(\Omega)\right),
\end{aligned}
$$

we can generate the complete set of $\mathrm{SO}(5)$ spherical harmonics and, from them, derive all required $\mathrm{SO}(5)$ Clebsch-Gordan coefficients [9, 5]. We also obtain [5] the $\mathrm{SO}(5)-$ reduced matrix elements

$$
\begin{aligned}
\left\langle\mathscr{Y}_{v_{3}}\left\|\hat{\mathscr{Y}}_{v_{2}}\right\| \mathscr{Y}_{v_{1}}\right\rangle= & \frac{1}{4 \pi} \frac{\left(\frac{\sigma}{2}+1\right) !}{\left(\frac{\sigma}{2}-v_{1}\right) !\left(\frac{\sigma}{2}-v_{2}\right) !\left(\frac{\sigma}{2}-v_{3}\right) !} \sqrt{\frac{\left(2 v_{1}+3\right)\left(2 v_{2}+3\right)}{\left(v_{3}+2\right)\left(v_{3}+1\right)}} \\
& \times \sqrt{\frac{(\sigma+4)\left(\sigma-2 v_{1}+1\right) !\left(\sigma-2 v_{2}+1\right) !\left(\sigma-2 v_{3}+1\right) !}{(\sigma+3) !}}
\end{aligned}
$$

where $\sigma=v_{1}+v_{2}+v_{3}$. Thus, Bohr model calculations are carried out quickly and easily.

Particularly informative are Bohr Hamiltonians are of the form

$$
\hat{H}(B, \lambda, \chi, \kappa)=-\frac{\nabla^{2}}{2 B}+V_{\lambda}(\beta, \gamma)
$$

with

$$
V_{\lambda}(\beta, \gamma)=\frac{1}{2} B\left[(1-2 \lambda) \beta^{2}+\lambda \beta^{4}\right]-\chi \beta \cos 3 \gamma+\kappa \cos ^{2} 3 \gamma
$$

For example, Fig. 3(a) shows the low energy-level spectrum and E2 transition rates for such a Hamiltonian with $\kappa=0$. The physical content of this spectrum is exposed by comparing it with results for the simpler Hamiltonian with potential

$$
V(\beta, \gamma) \approx V\left(\beta_{0}, \gamma_{0}\right)+\frac{1}{2} B \omega_{\beta}^{2}\left(\beta-\beta_{0}\right)^{2}+\frac{1}{2} B \beta_{0}^{2} \omega_{\gamma}^{2}\left(\gamma-\gamma_{0}\right)^{2}
$$

where $\beta_{0}=0$ and $\gamma_{0}=0$ are the values of $\beta$ and $\gamma$ at the potential minimum. With the additional assumption that the rotational motions of the model are slow relative to its $\beta$ and $\gamma$ vibrational modes there is an adiabatic decoupling of the rotational and vibrational degrees of freedom and the spectrum that emerges becomes as shown in Fig. 3(b). Thus, the relatively small differences between Figs. 3(a) and 3(b) can be attributed to SO(5) centrifugal coupling effects between the rotational and vibrational degrees of freedom that are included in Fig. 3(a) but not in 3(b).

The Bohr model and its many generalisations by Bohr, Mottelson, and numerous colleagues [10] has been enormously successful in the interpretation of a huge body of experimental data on collective motions. A particularly significant generalisation was the extension of the Bohr model to describe the dynamics of a nucleon coupled to an even-mass rotational nucleus [11]. This and other generalisations reveal how nucleons in a deformed mean field can form a rotor with many intrinsic degrees of freedom that can rotate adiabatically without undue disturbance of its intrinsic structure. Such generalisations are known as unified models. 


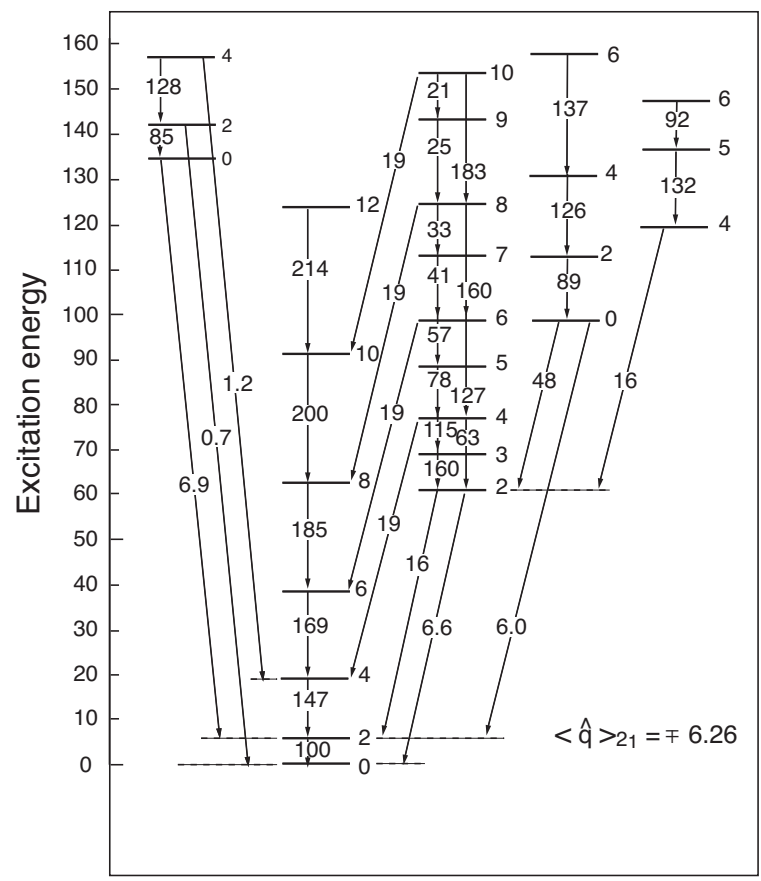

(a)

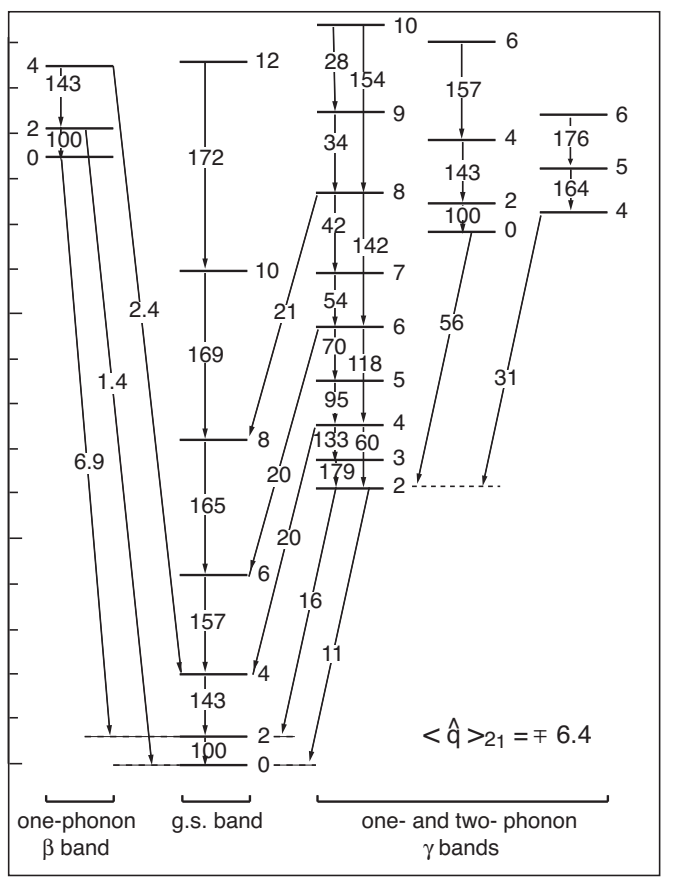

(b)

FIGURE 3. (a) The low-energy spectrum of the Hamiltonian $\hat{H}(B, \lambda, \chi, \kappa=0)$ of Equation (14) for $B=20, \lambda=1.5$, and $\chi= \pm 2.0$. Reduced E2 transition rates are shown in units for which $B\left(\mathrm{E} 2 ; 2_{1} \rightarrow\right.$ $\left.0_{1}\right)=100$. Energy levels are given in units such that the lowest $L=2$ state has energy $E_{2_{1}}=6$. (b) The corresponding results for the adiabatic Bohr model in the axially symmetric limit showing a ground-state band, a one-phonon beta vibrational band, and one- and two-phonon gamma vibrational bands. (Figure from [6])

\section{PROGRESSION TO A MICROSCOPIC COLLECTIVE MODEL}

In view of the successes of the Bohr and unified models, a great deal of effort has been expended in seeking to express collective dynamics in terms of microscopic nucleon coordinates. In retrospect, this is simple if one starts by replacing the surface shape coordinates $\left\{\alpha_{v}\right\}$ of the Bohr model by microscopic Cartesian monopole/quadrupole moments

$$
Q_{i j}=\sum_{n=1}^{A} x_{n i} x_{n j}, \quad i, j=1,2,3, n=1, \ldots, A .
$$

Time derivatives and corresponding momentum observables are then given by

$$
\dot{Q}_{i j}=\frac{d Q_{i j}}{d t}=\sum_{n}\left(\dot{x}_{n i} x_{n j}+x_{n i} \dot{x}_{n j}\right), \quad P_{i j}=M \dot{Q}_{i j}=\sum_{n}\left(p_{n i} x_{n j}+x_{n i} p_{n j}\right),
$$

where $M$ is the nucleon mass. These shape and momentum observables are now microscopic and close on a Lie algebra with commutation relations,

$$
\left[\hat{Q}_{i j}, \hat{P}_{k l}\right]=\mathrm{i} \hbar\left[\delta_{i l} \hat{Q}_{j k}+\delta_{i k} \hat{Q}_{j l}+\delta_{j l} \hat{Q}_{i k}+\delta_{j k} \hat{Q}_{i l}\right]
$$


This Lie algebra differs from that of the Bohr model. However, the subset of quadrupole moments defined in a spherical tensor basis by

$$
\begin{aligned}
& \hat{q}_{0}=\frac{1}{\sqrt{8}} \varepsilon\left(2 \hat{Q}_{11}-\hat{Q}_{22}-\hat{Q}_{33}\right), \\
& \hat{q}_{ \pm 1}=\sqrt{\frac{3}{4}} \varepsilon\left(\hat{Q}_{12} \pm \mathrm{i} \hat{Q}_{12}\right), \\
& \hat{q}_{ \pm 2}=\frac{\sqrt{3}}{4} \varepsilon\left(\hat{Q}_{22}-\hat{Q}_{33} \pm 2 \mathrm{i} \hat{Q}_{23}\right),
\end{aligned}
$$

have commutation relations with the corresponding quadrupole momenta that contract to those of the Bohr model when restricted to states of the Hilbert space of large monopole and relatively small quadrupole moments. For example, with the monopole moment defined by

$$
\hat{\mathfrak{M}}_{0}=\hat{Q}_{11}+\hat{Q}_{22}+\hat{Q}_{33}
$$

and $\varepsilon^{2}=1 /\left\langle\hat{\mathfrak{M}}_{0}\right\rangle$, it follows that

$$
\left[\hat{q}_{\mu}, \hat{p}^{v}\right]=\mathrm{i} \hbar \delta_{\mu}^{v}+0\left(\varepsilon^{2}\right) \rightarrow \mathrm{i} \hbar \delta_{\mu}^{v}, \quad \text { as } \varepsilon \rightarrow 0 .
$$

We refer the model with this SGA as the microscopic Bohr Model.

In spite of this result, this model fails to describe the low-energy rotational bands for which the Bohr model was introduced. This is because its momentum operators are the infinitesimal generators of irrotational-flow rotations for which the moments of inertia are much smaller than those needed to describe observed rotational bands. However, this limitation is overcome by enlarging the Lie algebra, spanned by $\left\{\hat{Q}_{i j}, \hat{P}_{i j}\right\}$, to include the angular momentum operators

$$
\hat{L}_{k}=\hbar \hat{L}_{i j}=\sum_{n}\left(\hat{x}_{n i} \hat{p}_{n j}-\hat{x}_{n j} \hat{p}_{n i}\right)
$$

The Lie algebra obtained is then isomorphic to a known $\mathrm{CM}(3)$ Lie algebra introduced by Weaver et al. [12].

The $\mathrm{CM}(3)$ model is richer than the Bohr model. Its six independent $\left\{\hat{Q}_{i j}\right\}$ observables characterize the size and quadrupole shape of a nucleus and, among its 9 momenta $\left\{\hat{L}_{i j}, \hat{P}_{i j}\right\}$, the angular momenta $\hat{L}_{i j}$ are infinitesimal generators of rigid-body rotations, the three components of $\hat{P}_{i j}$ with $i \neq j$ are infinitesimal generators of irrotational-flow rotations, and the three components $\hat{P}_{i i}$ are infinitesimal generators of diagonal shape scale transformations [13]. Combinations of these momentum operators also generate intrinsic current circulations as illustrated in the third row of Fig. 4.

The unitary irreps of $\mathrm{CM}(3)$ were determined by Rosensteel [14] with the intention of developing a microscopic theory of collective motion. However, two obstacles were encountered: one was that, while the many-nucleon kinetic energy has a simple known expression, $T=\frac{1}{2 m} \sum_{n i} \hat{p}_{n i}^{2}$, its $\mathrm{CM}(3)$ collective model component (derived for example in [15]) proved to be excessively complicated; another was that the $\mathrm{CM}(3)$ representations did not relate in a natural way to the nuclear shell model. The way around both obstacles was simply to augment the $\mathrm{CM}(3)$ algebra further so that it included the manynucleon kinetic energy. The result was the much more powerful symplectic model. 


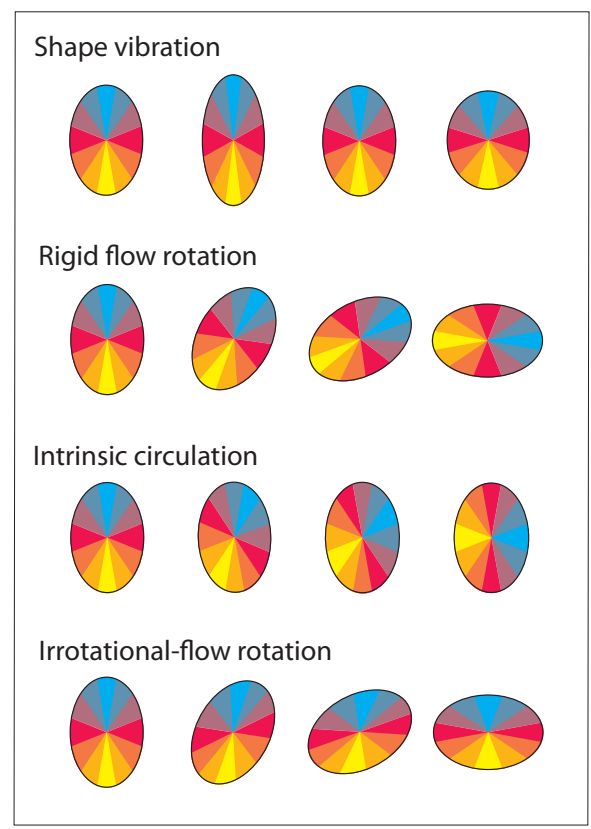

FIGURE 4. Collective flows generated by $\mathrm{CM}(3)$ transformations

\section{THE SYMPLECTIC MODEL}

The symplectic model $[16,17]$ is an algebraic model whose dynamical group $\operatorname{Sp}(3, \mathrm{R})$ comprises all $n$-independent linear canonical transformations of the single-particle phase-space observables

$$
\hat{x}_{n i} \rightarrow \sum_{j}\left(a_{i j} \hat{x}_{n j}+b_{i j} \hat{p}_{n j}\right), \quad \hat{p}_{n i} \rightarrow \sum_{j}\left(c_{i j} \hat{x}_{n j}+d_{i j} \hat{p}_{n j}\right)
$$

that preserve the commutation relations $\left[\hat{x}_{n i}, \hat{p}_{m j}\right]=\mathrm{i} \hbar \delta_{m, n} \delta_{i, j}$. Its spectrum generating algebra is spanned by the elements of the $\mathrm{CM}(3)$ Lie algebra

$$
\hat{Q}_{i j}=\sum_{n=1}^{A} \hat{x}_{n i} \hat{x}_{n j}, \quad \hat{P}_{i j}=\sum_{n}\left(\hat{p}_{n i} \hat{x}_{n j}+\hat{x}_{n i} \hat{p}_{n j}\right), \quad \hbar \hat{L}_{i j}=\sum_{n}\left(\hat{x}_{n i} \hat{p}_{n j}-\hat{x}_{n j} \hat{p}_{n i}\right),
$$

plus the bilinear momentum operators

$$
\hat{K}_{i j}=\sum_{n} \hat{p}_{n i} \hat{p}_{n j}
$$

Adding the $\hat{K}_{i j}$ operators to the $\mathrm{CM}(3)$ algebra is invaluable for many reasons:

(i) The representations of $\mathrm{Sp}(3, \mathrm{R})$ are easier to handle than those of $\mathrm{CM}(3)$.

(ii) The many-nucleon kinetic energy and harmonic-oscillator Hamiltonians

$$
\sum_{n=1}^{A} \sum_{i=1}^{3} \frac{1}{2 M} \hat{p}_{n i}^{2}, \quad \hat{H}_{\mathrm{DHO}}^{(A)}=\sum_{n=1}^{A}\left[\frac{1}{2 M} \hat{\mathbf{p}}_{n}^{2}+\frac{1}{2} M\left(\omega_{1}^{2} \hat{x}_{n 1}^{2}+\omega_{2}^{2} \hat{x}_{n 2}^{2}+\omega_{3}^{2} \hat{x}_{n 3}^{2}\right)\right],
$$


where $M$ is the nucleon mass, are elements of the $\mathrm{Sp}(3, \mathrm{R})$ Lie algebra for all values of $\omega_{1}, \omega_{2}, \omega_{3}$. As a result, the symplectic model is compatible with the harmonic oscillator shell model and with unified collective models such as the Nilsson model.

(iii) $\mathrm{Sp}(3, \mathrm{R})$ representation theory makes it possible to diagonalize a collective model Hamiltonian

$$
\hat{H}=\frac{1}{2 M} \sum_{i} \hat{K}_{i i}+V(Q),
$$

in a basis of shell-model states.

(iv) The group, $\mathrm{Sp}(3, \mathrm{R})$, contains important subgroup chains

$$
\begin{aligned}
& \mathrm{Sp}(3, \mathrm{R}) \supset \mathrm{CM}(3) \supset \mathrm{ROT}(3) \supset \mathrm{SO}(3), \\
& \mathrm{Sp}(3, \mathrm{R}) \supset \mathrm{U}(3) \supset \mathrm{SU}(3) \supset \mathrm{SO}(3),
\end{aligned}
$$

where ROT(3) is a dynamical group for a rigid-rotor model and SU(3) is the dynamical group of Elliott's SU(3) model. The first chain reflects its content in terms of the dynamical flows of a quantum fluid. The latter chain defines an optimal shell-model coupling scheme for a shell-model description of collective states in nuclei.

These properties are all you could wish for from a microscopic collective model.

\subsection{The $\operatorname{Sp}(3, R)$ algebra in a $U(3)$ basis}

The $\mathrm{U}(3) \subset \mathrm{Sp}(3, \mathrm{R})$ subalgebra consists of all elements of the $\operatorname{Sp}(3, \mathbb{R})$ Lie algebra that commute with the spherical harmonic-oscillator Hamiltonian

$$
\hat{H}_{\mathrm{SHO}}^{(A)}=\sum_{n=1}^{A}\left[\frac{1}{2 M} \hat{\mathbf{p}}_{n}^{2}+\frac{1}{2} M \omega^{2}\left(\hat{x}_{n 1}^{2}+\hat{x}_{n 2}^{2}+\hat{x}_{n 3}^{2}\right)\right],
$$

where $\omega$ is fixed to get the mean-square radius of a nucleus at its observed value.

To identify this subalgebra, express the $\hat{x}_{n i}$ and $\hat{p}_{n i}$ observables in terms of harmonic oscillator raising and lowering operators

$$
\hat{x}_{n i}=\frac{1}{\sqrt{2} a}\left(c_{n i}^{\dagger}+c_{n i}\right), \quad \hat{p}_{n i}=\mathrm{i} \hbar \frac{a}{\sqrt{2}}\left(c_{n i}^{\dagger}-c_{n i}\right),
$$

where $a=\sqrt{M \omega / \hbar}$ is the harmonic oscillator unit of inverse length. These operators satisfy the commutation relations

$$
\left[c_{n i}, c_{m j}^{\dagger}\right]=\delta_{m, n} \delta_{i, j}, \quad\left[\hat{H}_{\mathrm{SHO}}^{(A)}, c_{n i}^{\dagger}\right]=\hbar \omega c_{n i}^{\dagger}, \quad\left[\hat{H}_{\mathrm{SHO}}^{(A)}, c_{n i}\right]=-\hbar \omega c_{n i} .
$$

Expansion of the $\operatorname{Sp}(3, R)$ operators in terms of them gives

$$
a^{2} \hat{Q}_{i j}=\hat{\mathscr{Q}}_{i j}+\hat{A}_{i j}+\hat{B}_{i j}
$$




$$
\begin{aligned}
& \hat{P}_{i j}=2 \mathrm{i} \hbar\left(\hat{A}_{i j}-\hat{B}_{i j}\right), \\
& \frac{1}{a^{2}} \hat{K}_{i j}=\hbar^{2}\left(\hat{\mathscr{Q}}_{i j}-\hat{A}_{i j}-\hat{B}_{i j}\right), \\
& \hat{L}_{i j}=-\mathrm{i}\left(\hat{C}_{i j}-\hat{C}_{j i}\right),
\end{aligned}
$$

where

$$
\begin{aligned}
& \hat{A}_{i j}=\hat{A}_{j i}=\frac{1}{2} \sum_{n} c_{n i}^{\dagger} c_{n j}^{\dagger}, \quad \hat{B}_{i j}=\hat{B}_{j i}=\frac{1}{2} \sum_{n} c_{n i} c_{n j}, \\
& \hat{C}_{i j}=\sum_{n}\left(c_{n i}^{\dagger} c_{n j}+\frac{1}{2}\right), \quad \hat{\mathscr{Q}}_{i j}=\frac{1}{2}\left(\hat{C}_{i j}+\hat{C}_{j i}\right),
\end{aligned}
$$

satisfy the commutation relations

$$
\begin{aligned}
& {\left[\hat{H}_{\mathrm{SHO}}^{(A)}, \hat{\mathscr{Q}}_{i j}\right]=0, \quad\left[\hat{H}_{\mathrm{SHO}}^{(A)}, \hat{L}_{i j}\right]=0,} \\
& {\left[\hat{H}_{\mathrm{SHO}}^{(A)}, \hat{A}_{i j}\right]=2 \hbar \omega \hat{A}_{i j}, \quad\left[\hat{H}_{\mathrm{SHO}}^{(A)}, \hat{B}_{i j}\right]=-2 \hbar \omega \hat{B}_{i j} .}
\end{aligned}
$$

Thus, the U(3) subalgebra is spanned by the subset of operators $\left\{\hat{\mathscr{Q}}_{i j}, \hat{L}_{i j}\right\}$ that commute with $\hat{H}_{\mathrm{SHO}}^{(A)}$, and the operators $\left\{\hat{A}_{i j}\right\}$ and $\left\{\hat{B}_{i j}\right\}$ are, respectively, $\pm 2 \hbar \omega$ raising and lowering operators for representations of the $\operatorname{sp}(3, \mathbb{R}))$ algebra. The $L=0$ combination $\hat{A}_{0}=\sqrt{\frac{2}{3}} \sum_{i} \hat{A}_{i i}$ is the creation operator for a $2 \hbar \omega$ giant-monopole excitation and the five $L=2$ combinations $\left\{\hat{A}_{2 v} ; v=0, \pm 1, \pm 2\right\}$ are creation operators for $2 \hbar \omega$ giantquadrupole excitations. Together, these six raising operators transform as the $L=0$ and 2 components of an SU(3) (20) tensor (see Exercise ??).

\subsection{Irreducible representations (irreps) of $S p(3, R)$ in a $U(3)$ basis}

The $\operatorname{Sp}(3, \mathrm{R})$ Lie alglebra is non-compact and, like the Lie algebra of the Bohr model, it has only infinite-dimensional unitary irreps. In the space of the nuclear shell model it has irreps with lowest weights, but none with highest weights. Thus, irreps of $\operatorname{Sp}(3, R)$ are constructed within the shell-model space by first defining their lowest-weight states which are also conveniently defined as U(3) highest-weight states.

A U(3) highest-weight state is labelled by three quantum numbers $|N(\lambda \mu)\rangle$ and satisfies the equations

$$
\begin{aligned}
& \hat{C}_{i i}|N(\lambda \mu)\rangle=N_{i}|N(\lambda \mu)\rangle, \quad i=1,2,3, \\
& \left(\hat{C}_{11}+\hat{C}_{22}+\hat{C}_{33}\right)|N(\lambda \mu)\rangle=N|N(\lambda \mu)\rangle, \\
& \left(\hat{C}_{11}-\hat{C}_{22}\right)|N(\lambda \mu)\rangle=\lambda|N(\lambda \mu)\rangle, \\
& \left(\hat{C}_{22}-\hat{C}_{33}\right)|N(\lambda \mu)\rangle=\mu|N(\lambda \mu)\rangle, \\
& \hat{C}_{i j}|N(\lambda \mu)\rangle=0, \quad \forall 1 \leq i<j \leq 3,
\end{aligned}
$$

with

$$
N=N_{1}+N_{2}+N_{3}, \quad \lambda=N_{1}-N_{2}, \quad \mu=N_{2}-N_{3} .
$$

An $\operatorname{Sp}(3, \mathrm{R})$ lowest-weight state $\left|N_{0}\left(\lambda_{0} \mu_{0}\right)\right\rangle$ is a U(3) highest-weight state that is also annihilated by the giant-resonance lowering operators, i.e.,

$$
\hat{B}_{i j}\left|N_{0}\left(\lambda_{0} \mu_{0}\right)\right\rangle=0, \quad \forall 1 \leq i \leq j \leq 3
$$


A U(3) irrep with highest-weight state $|N(\lambda \mu)\rangle$ is denoted by $\{N(\lambda \mu)\}$ and an $\operatorname{Sp}(3, \mathrm{R})$ irrep with lowest-weight state $\left|N_{0}\left(\lambda_{0} \mu_{0}\right)\right\rangle$ is denoted by $\left\langle N_{0}\left(\lambda_{0} \mu_{0}\right)\right\rangle$. A U(3) irrep whose highest-weight state is also an $\operatorname{Sp}(3, \mathrm{R})$ lowest-weight state is said to be a lowest-grade $\mathrm{U}(3)$ irrep for that $\mathrm{Sp}(3, \mathrm{R})$ irrep. It follows that basis states for an $\mathrm{Sp}(3, \mathrm{R})$ irrep are obtained by augmenting a basis for a lowest-grade $U(3)$ irrep by addition of an infinite set of giant-resonance excitations generated by the $\left\{\hat{A}_{i j}\right\}$ raising operators.

There are four classes of U(3) irreps: $\{N(00)\},\{N(\lambda 0)\},\{N(0 \lambda)\}$ and $\{N(\lambda \mu)\}$. The $\{N(00)\}$ irreps are one-dimensional and contain a single $L=0$ state. The $\{N(0 \lambda)\}$ and $\{N(\lambda 0)\}$ irreps are contragredient to one another; they have common spectra of angular momentum states, given by

$$
L=\lambda, \lambda-2, \lambda-4, \ldots, 1 \text { or } 0,
$$

and differ only in that their quadrupole matrix elements are of opposite sign. A generic $\mathrm{U}(3)$ irrep $\{N(\lambda \mu)\}$ contains a single $\mathrm{SU}(3)$ irrep $(\lambda \mu)$ and has a spectrum of $\mathrm{SO}(3)$ angular-momentum states given by the $\mathrm{SU}(3) \rightarrow \mathrm{SO}(3)$ branching rule

$$
\begin{array}{rrr}
(\lambda \mu) \mapsto & L=\lambda, \lambda-2, \ldots, 1 \text { or } 0, & \text { when } K=0, \\
& \mapsto \quad L=K, K+1, \ldots, K+\lambda, & \text { when } K \neq 0, \\
& & \text { for } K=\mu, \mu-2, \mu-4, \ldots, 1 \text { or } 0 .
\end{array}
$$

At this point it is useful to recall that $\mathrm{SU}(3)$ irreps closely resemble truncated irreps of a rotor. In fact, for values of $\lambda$ and/or $\mu$ large compared to the angular momenta of the states of interest, the properties of the SU(3) irreps $(\lambda 0)$ and $(0 \lambda)$ approach those of prolate and oblate rigid rotors, respectively, whereas the properties of a generic SU(3) irrep $(\lambda \mu)$ approach those of a triaxial rigid rotor. Thus, an SU(3) model was introduced by Elliott [2] as a first step towards a shell-model description of rotational states in light nuclei. Typical spectra for SU(3) model irreps are shown in Fig, 5.

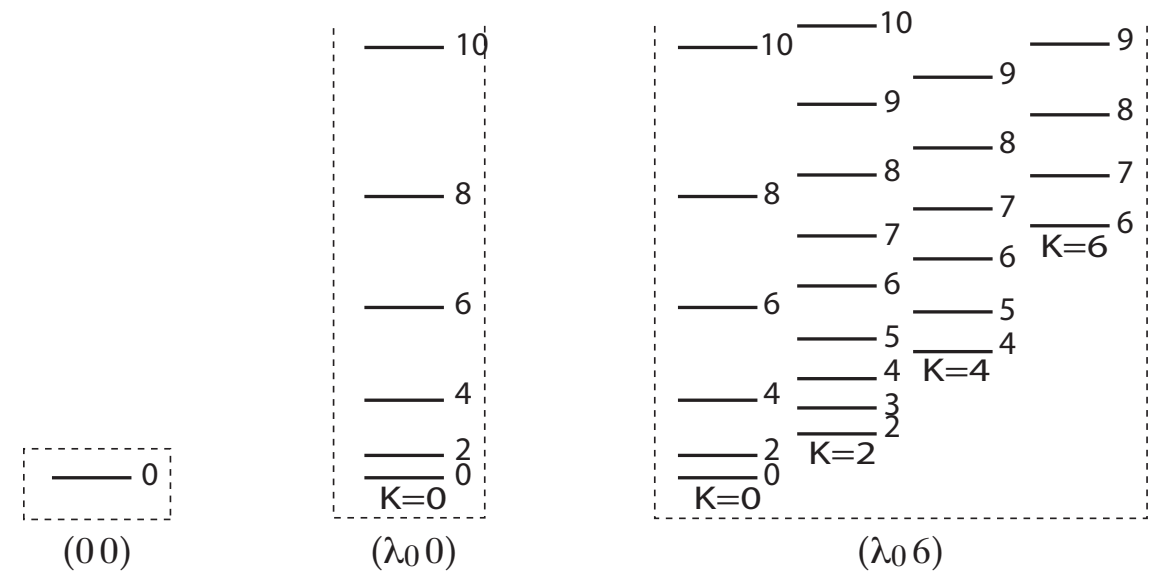

FIGURE 5. Typical SU(3) model spectra for the lowest-energy states of three irreps of even values of $\lambda_{0}$. The $(00)$ irrep contains a single state.

The energy spectrum of basis states for an $\operatorname{Sp}(3, \mathrm{R})$ irrep, with respect to the spherical harmonic-oscillator Hamiltonian, Eq. (27), is now obtained as follows. The states of 
a lowest-grade $\mathrm{U}(3)$ irrep $\left\{\left\langle N_{0}\left(\lambda_{0} \mu_{0}\right)\right\rangle\right\}$ all have the common harmonic-oscillator energy $N_{0} \hbar \omega$. One-phonon giant-resonance states appear at an energy of $\left(N_{0}+2\right) \hbar \omega$ and because the giant-resonance raising operators are components of an SU(3) (20) tensor, they generate the states of a generally reducible $S U(3)$ representation given by the $S U(3)$ tensor product $\left(\lambda_{0} \mu_{0}\right) \otimes(20)$. The spectra of basis states are illustrated in Fig. 6.

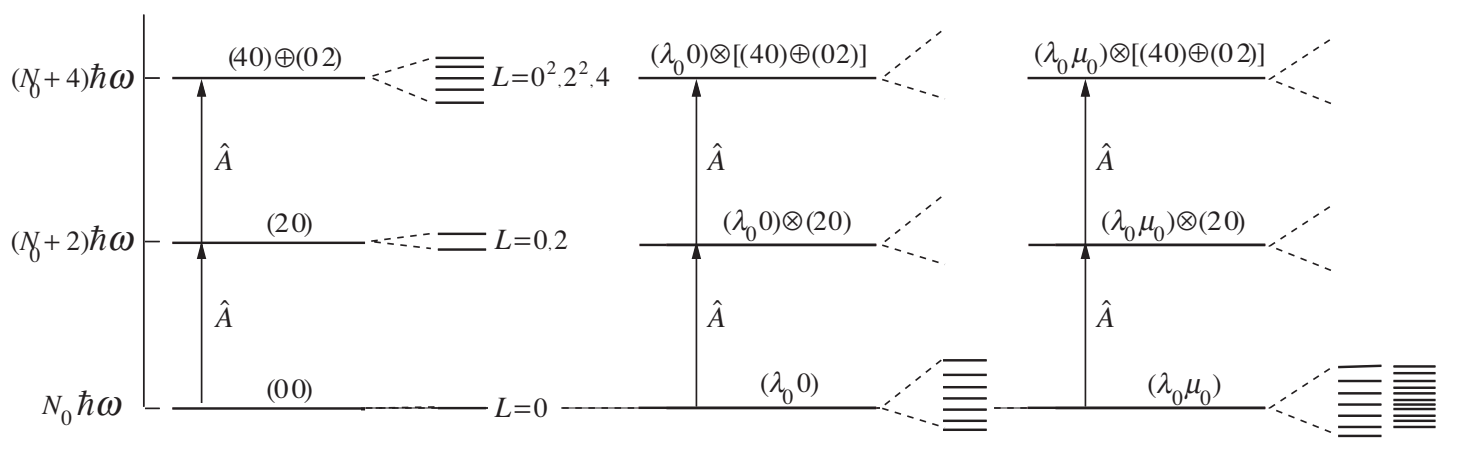
(a) $\left\langle N_{0}(00)\right\rangle$
b) $\left\langle N_{0}\left(\lambda_{0} 0\right)\right\rangle$
(c) $\left\langle N_{0}\left(\lambda_{0} \mu_{0}\right)\right\rangle$

FIGURE 6. Low basis states for $\operatorname{Sp}(3, \mathbb{R})$ irreps ordered by their spherical harmonic oscillator energies: (a) for an $\left\langle N_{0}(00)\right\rangle$ irrep; (b) for a $\left\langle N_{0}\left(\lambda_{0} 0\right)\right\rangle$ irrep; (c) for a generic $\left\langle N_{0}\left(\lambda_{0} \mu_{0}\right)\right\rangle$ irrep.

It is of interest to note that the spectrum of states for an $\left\langle N_{0}(00)\right\rangle$ irrep is in 1-1 correspondence with that of a 6-dimensional harmonic oscillator. In fact, they are a basis for an irrep of the microscopic Bohr model, spanned by the $\left\{\hat{Q}_{i j}, \hat{P}_{i j}\right\}$ operators of Eq. (17), which comprises giant monopole and quadrupole harmonic vibrational states with irrotational-flow mass parameters. More generally, the states of an $\mathrm{Sp}(3, \mathrm{R})$ irrep are in 1-1 correspondence with the states of such a Bohr model coupled to a non-trivial SU(3) irrep. This is illustrated pictorially in Fig. 7. Moreover, it has been shown [18, 19, 20]

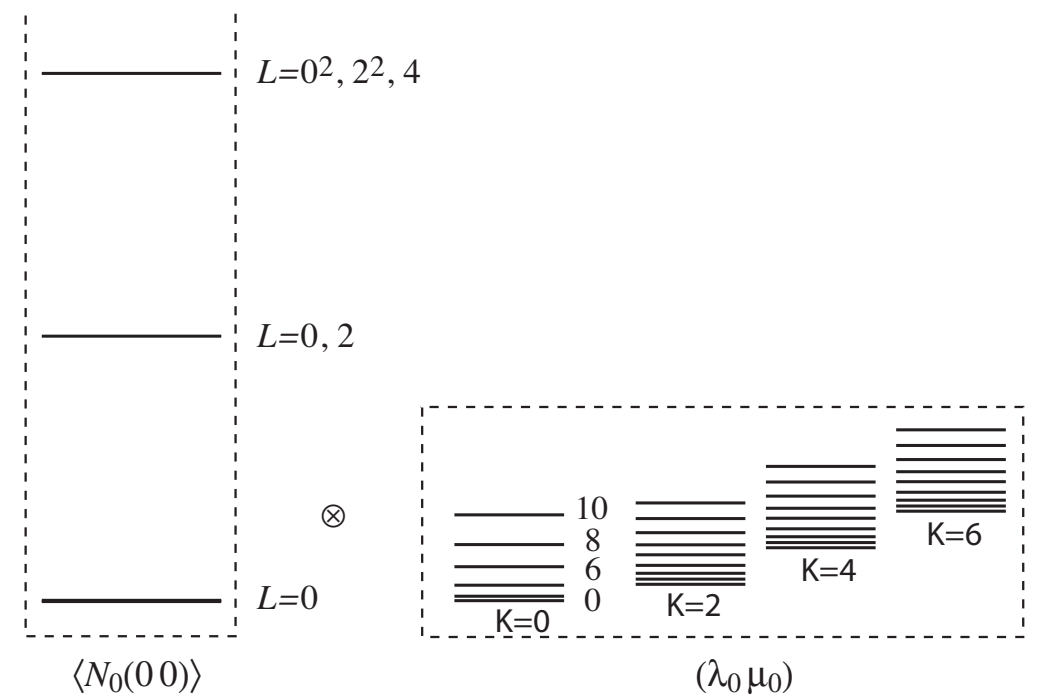

FIGURE 7. Basis states for the $\mathrm{Sp}(3, \mathrm{R})$ model are in 1-1 corresondence with a coupled product of the Bohr model (realised in terms of giant monopole-quadrupole resonance states with irrotational flows) and an SU(3) model. 
that, in the limit of large quantum numbers, the $\operatorname{Sp}(3, \mathrm{R})$ model contracts to the coupled product of a Bohr vibrational model and a rotor model. This is the so-called coupled rotor-vibrator limit of the symplectic model.

A simple prediction that follows, without a calculation, from the observation that the underlying microscopic description of monopole/quadrupole rotational and vibrational dynamics is provided by the symplectic model, is that this model contains no low-energy $\beta$ or $\gamma$ vibrational bands [21]. It does exhibit excited $K=2$ bands but these are more of a triaxial rotor than of a vibrational nature. Excited $K=0$ bands (for even-even) nuclei do occur in the model but are associated with an excited $\operatorname{Sp}(3, \mathrm{R})$ irrep. With mixing of $\operatorname{Sp}(3, \mathrm{R})$ irreps, such an excited band can exhibit properties associated with a $\beta$ band. However, the occurrence of such bands is much more appropriately described in terms of band mixing and as a departure from the underlying symmetry rather than as a part of it. The study of such $K=0$ bands and the E2 transitions between them is therefore expected to provide valuable information on the mixing of $\operatorname{Sp}(3, \mathrm{R})$ irreps in even-even nuclei (see, e.g., [22]).

\subsection{A model $S p(3, R)$ calculation}

Figure 8 shows the energy levels and reduced E2 transition rates for a symplectic model calculation [23] for an $\operatorname{Sp}(3, \mathrm{R})$ irrep $\langle 826.5(78,0)\rangle$ with the two-parameter Hamiltonian

$$
\hat{H}=\hat{H}_{\mathrm{SHO}}^{(166)}+\chi\left(\hat{Q} \cdot \hat{Q}+\frac{\varepsilon}{\hat{Q} \cdot \hat{Q}}\right) .
$$

The irrep $\langle 826.5(78,0)\rangle$ was chosen to be appropriate for ${ }^{166} \mathrm{Er}$ on the basis of experimental data [24] (as discussed below). The two parameters $\varepsilon$ and $\chi$ of this Hamiltonian are naturally defined as follows: $\varepsilon$ can be adjusted so that the potential energy component of the Hamiltonian (42) has a minimum at the deformation determined from experimental E2 transition rates; the strength parameter $\chi$ is then determined by the selfconsistency condition that the density distribution for the wave function has the same quadrupole shape as that of the potential. For the results shown, the simpler procedure was followed of adjusting $\varepsilon$ as mentioned above to accord with the observed deformation, and adjusting $\chi$ to give the observed energy of the $2_{1}$ state. The close agreement of the results, especially for states of lower angular momentum is very satisfying because it means that the symplectic model is describing the physics of a rotational band of states with realistic moments of inertia that emerge naturally without any adjustment of the many-nucleon kinetic energy, e.g., by introducing an effective nucleon mass.

The fact that the symplectic model agrees closely with a two-parameter rotor model is also satisfying because, while the rotational character of the states described is presumed in the rotor model, no such assumption is made in the symplectic model which has many more degrees of freedom. In fact, it is apparent that the experimental energy levels exhibit a degree of centrifugal stretching that is not admitted in the rigid-rotor model. Thus, the rotor model interpretation of the symplectic model results suggests that a better agreement with the higher energy levels could have been obtained in the symplectic model with a potential energy having a less rigidly-defined minimum. The agreement 


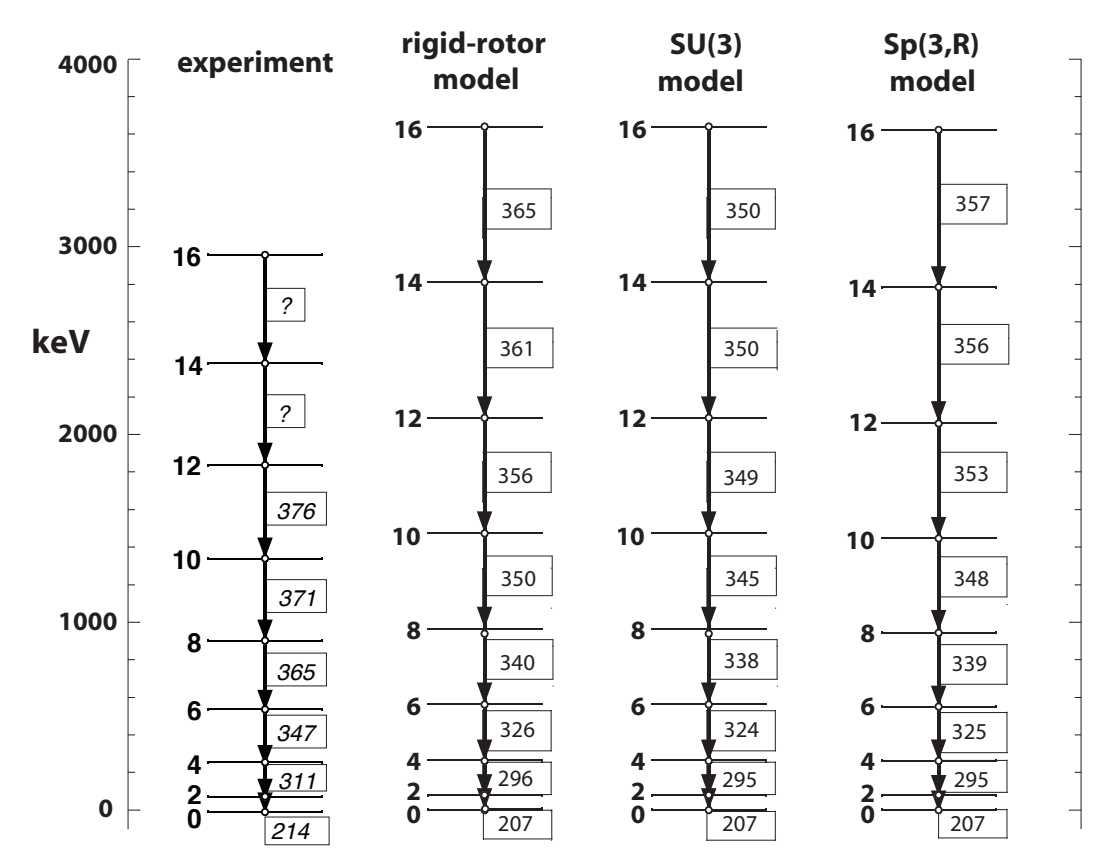

FIGURE 8. Low-energy states of ${ }^{166} \mathrm{Er}$ calculated in an $\mathrm{Sp}(3, \mathrm{R})$ irrep $\langle 826.5(78,0)\rangle$ for the Hamiltonian (42). For comparison the spectra calculated in the rotor and SU(3) models are also shown. The boxed numbers are B(E2)'s in W.u.

between the two-parameter SU(3) and rigid-rotor models is not surprising, in view of the contraction, noted above, of the SU(3) model to the rotor model for large-dimensional representations. However, the relationship between the symplectic model and these two models is important for understanding the microscopic structure of rotational states and for understanding how the seeds of nuclear rotations can be found within the framework of a shell-model coupling scheme.

\section{THE SHELL-MODEL THEORY OF DEFORMED NUCLEI}

Because the many-nucleon Hilbert space is infinite dimensional, many-nucleon calculations must necessarily be restricted to finite-dimensional subspaces. Thus, to obtain meaningful results, it is necessary to choose the finite-dimensional subspaces such that they have significant relationships with the physical states they purport to describe.

The standard shell model is based on the presumption that the dominant forces on a nucleon in the nucleus are generated by a spherically symmetric mean-field with spin-orbit interactions. Thus, a hierarchy of standard shell model states is defined by a partially-ordered basis of independent-particle model states. In this model, manynucleon subspaces are naturally defined by specification of occupied and unoccupied single-particle states and a complementary valence space of partially-occupied states. Such subspaces can be ordered by their summed independent-particle-model energies. Which subspace one chooses for a specific purpose obviously depends on one's objectives and computational resources. However, a common practice is to choose a subspace 
that is as large as can be handled and, apart from symmetry constraints (such as parity), is one for which all independent-particle states of energy below those of the valence space are occupied.

In practice, the mean field of the shell model is commonly replaced by a spherical harmonic oscillator potential. This makes it possible to capitalise on the rich variety of symmetries associated with the harmonic oscillator, which greatly facilitate the execution of complex calculations. It must be remembered though that the purpose of the independent-particle potential is to define basis states for a many-nucleon calculation and the better the basis the fewer basis states are needed to obtain results to a given level of accuracy. Except for the radial tails of the wave functions, which are important in reaction theory but less important for the calculation of bound states, a spherical harmonic oscillator does a reasonably good job of approximating the mean field for spherical nuclei and the inadequacies of the bases that it provides are more than compensated by the many benefits resulting from the harmonic oscillator symmetries.

A concern addressed in these lectures is that, while the standard spherical harmonic oscillator shell model is very successful for the description of spherical nuclei, it fails badly in the description of strongly-deformed nuclei. This is revealed by considering which $\operatorname{Sp}(3, \mathrm{R})$ irreps are appropriate for the description of rotational bands in nuclei and finding that they lie in shell-model subspaces that are often far above those that would normally be included in the valence spaces of the spherical harmonic-oscillator shell model. It is insightful to consider this concern from a mean-field perspective.

\subsection{Geometrical and mean-field perspectives}

Loosely speaking, mean-field theory can be regarded as quantum mechanics constrained to an orbit of a dynamical group that is isomorphic to a classical phase space. Thus, mean-field theory provides an interface between classical and quantum mechanics and enables one to obtain valuable classical insights into the quantum world. For example, in quantum mechincs, the ground state of a Hamiltonian is contained in an irrep of a dynamical group for that Hamiltonian. In mean-field theory, a classical ground state is an equilibrium state given by the minimal-energy state on the classical phase space. This minimum energy is then an upper bound on the quantum-mechanical ground state energy. Thus, it is meaningful to order $\operatorname{Sp}(3, \mathrm{R})$ irreps by their minimal energy mean-field states.

Let $|\sigma\rangle=\left|N_{0}\left(\lambda_{0} \mu_{0}\right)\right\rangle$ denote a lowest-weight state for an $\operatorname{Sp}(3, \mathrm{R})$ irrep and let $\hat{T}(g)$ denote a unitary transformation of the states of this irrep by an element $g \in \operatorname{Sp}(3, R)$. The group orbit is then the manifold of coherent states

$$
\mathscr{M}=\{|\sigma(g)\rangle=\hat{T}(g)|\sigma\rangle ; g \in \operatorname{Sp}(3, \mathrm{R})\},
$$

which is isomorphic to the classical phase space for the irrep. We now claim that the real submanifold of zero-momentum states of this phase space is the set

$$
\mathscr{R}=\left\{|\sigma(g)\rangle=\hat{T}(g)|\sigma\rangle ; g \in \mathrm{GL}_{+}(3, \mathrm{R})\right\},
$$


where $\mathrm{GL}_{+}(3, \mathrm{R})$ is the connected subgroup of real $3 \times 3$ matrices with positive determinant. To see this, first observe that the phase space $\mathscr{M}$ has $\operatorname{dimension} \operatorname{dim}(\mathscr{M})=18$; this follows because the $\mathrm{Sp}(3, \mathrm{R})$ Lie algebra is of dimension 21 but, as shown by Eqs. (34) (36), three elements of this Lie algebra, namely $\hat{C}_{11}, \hat{C}_{22}$ and $\hat{C}_{33}$, leave the state $|\sigma\rangle$ invariant. Next observe that the nine linearly-independent momentum operators $\hat{P}_{i j}=\hat{P}_{j i}$ and $\hat{L}_{i j}=-\hat{L}_{j i}$ are the infinitesimal generators of the real subspace $\mathscr{R} \subset \mathscr{M}$ and that they span a Lie algebra isomorphic to that of $\mathrm{GL}_{+}(3, \mathrm{R})$.

We now make use of the fact that a general linear matrix $g \in \mathrm{GL}_{+}(3, \mathrm{R})$ can be expressed as a matrix product

$$
g=\Omega \mathbf{d} \Omega^{\prime},
$$

where $\Omega$ and $\Omega^{\prime}$ are $\mathrm{SO}(3)$ matrices and $\mathbf{d}=\operatorname{diag}\left(d_{1}, d_{2}, d_{3}\right)$ is a real diagonal matrix with positive entries. Thus, in the unitary representation $\hat{T}$, we can express the meanfield states of $\mathscr{R}$ in the form

$$
\left|\sigma\left(\Omega d \Omega^{\prime}\right)\right\rangle=\hat{R}(\Omega) \hat{U}(\mathbf{d}) \hat{R}\left(\Omega^{\prime}\right)|\sigma\rangle
$$

where $\hat{R}(\Omega)$ is a rotation/inversion operator and $\hat{U}(\mathbf{d})$ is a unitary representation of the scale transformation $\left\{x_{n i} \rightarrow d_{i} x_{n i}, i=1,2,3\right\}$.

The sequence of transformations $\hat{R}(\Omega) \hat{U}(\mathbf{d}) \hat{R}\left(\Omega^{\prime}\right)$ of the $\operatorname{Sp}(3, \mathrm{R})$ lowest-weight state $|\sigma\rangle$ is represented pictorially in Fig. 9 for two $\operatorname{Sp}(3, \mathrm{R})$ irreps, $\left\langle N_{0}(00)\right\rangle$ and a generic irrep $\left\langle N_{0}\left(\lambda_{0} \mu_{0}\right)\right\rangle$. For the purpose of this representation, it is convenient to make use

(i) $\left\langle N_{0}(00)\right\rangle$
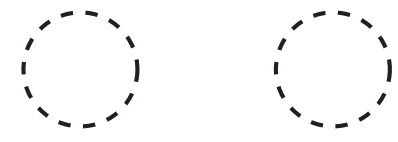

(ii)

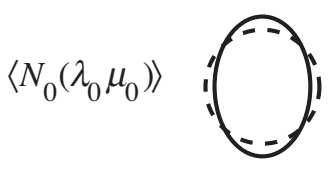

(a)

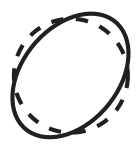

(b)
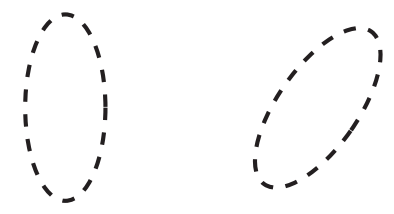

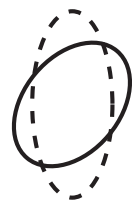

(c)

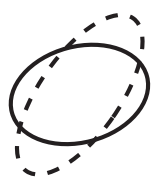

(d)

$|\sigma\rangle \quad \hat{R}\left(\Omega^{\prime}\right)|\sigma\rangle \quad \hat{U}(d) \hat{R}\left(\Omega^{\prime}\right)|\sigma\rangle \quad \hat{R}(\Omega) \hat{U}(d) \hat{R}\left(\Omega^{\prime}\right)|\sigma\rangle$

$\mathrm{SU}(3)$ rotations $\quad \mathrm{SU}(3)+\mathrm{GR}$ rotations

FIGURE 9. A geometrical perspective of the nine dynamical degrees of freedom of the symplectic model which reduce to six for a spherical irrep $\left\langle N_{0}(00)\right\rangle$ wherein the three $\mathrm{SU}(3)$ rotational degrees of freedom are suppressed; see the text for an explanation of this figure.

of the 1-1 correspondence between the basis states of the symplectic model and those of a coupled Bohr (giant-resonance) model and an SU(3) model as depicted in Fig. 7. The states of the manifold $\mathscr{R}$ can then be represented by a pair of ellipsoids: one of fixed shape but arbitrary orientation, representing the three rotational degrees of freedom of the SU(3) rotor; and one with a full range of ellipsoidal shapes and orientations, representing the six degrees of freedom of the Bohr model. The states of the SU(3) rotor are drawn in the figure with solid lines while those for the giant-resonance are drawn 
with dashed lines. Recall, however, that for the $\operatorname{Sp}(3, \mathrm{R})$ irrep $\left\langle N_{0}(00)\right\rangle$ the lowest-grade $\mathrm{SU}(3)$ irrep is the trivial identity representation. For this irrep only the giant-resonance degrees of freedom survive and the lowest-weight state $\left\langle N_{0}(00)\right\rangle$ is the giant-resonance vacuum state which has a spherical density distribution as shown in row (i) column (a) of the figure. For a generic $\operatorname{Sp}(3, \mathrm{R})$ irrep the lowest-weight state $|\sigma\rangle$ is again a giantresonance vacuum state. But it is also the highest-weight state for a non-trivial SU(3) irrep with a non-spherical density distribution, as shown in row (ii) column (a).

Because the action of the rotation operator $\hat{R}\left(\Omega^{\prime}\right)$ on the lowest-weight state $|\sigma\rangle$ does not excite any giant-resonance states, its effect is simply to reorient the SU(3) ellipsoidal density distribution. Conversely, the transformation $\hat{U}(\mathbf{d})$, shown in (c), is a diagonal scale transformation which excites giant resonance degrees of freedom and changes the spherical shape of the giant-resonance vacuum state to a more general ellipsoidal shape, with its principal axes aligned along the space-fixed axes, but leaves the SU(3) degrees of freedom alone. Finally the last rotation $\hat{R}(\Omega)$, shown in (d) rotates both the $\mathrm{SU}(3)$ and giant-resonance ellipsoids. The resulting shape of the nucleus, as represented by Fig. 9(d), is now a composition of the two ellipsoids shown in which that corresponding to the SU(3) lowest grade ellipsoid is scaled in a manner defined by the GR ellipsoid.

\subsection{The minimal-energy mean-field state for an $\operatorname{Sp}(3, R)$ irrep}

To derive the minimal-energy mean-field state for an $\operatorname{Sp}(3, R)$ irrep, one would ideally make a variational calculation, analogous to a Hartree-Fock calculation, on the phase space of $\operatorname{Sp}(3, \mathrm{R})$ coherent states defined by Eq. (43). However, it is possible to assess what the result would be on the basis of a simple self-consistency argument. First observe that, for a time-reversal-invariant Hamiltonian, the minimal-energy state should be one with vanishing expectation values of its momentum operators. Thus, it should be the state in the set $\left\{|\sigma(g)\rangle, g \in \mathrm{GL}_{+}(3, \mathrm{R})\right\}$ for which

$$
E\left(\Omega \mathbf{d} \Omega^{\prime}\right)=\left\langle\sigma\left(\Omega \mathbf{d} \Omega^{\prime}\right)|\hat{H}| \sigma\left(\Omega \mathbf{d} \Omega^{\prime}\right)\right\rangle
$$

is a minimum for the given Hamiltonian.

The next observation is that, for a rotationally-invariant Hamiltonian, the energy is independent of $\Omega$. We also suppose that, because the giant-resonance contribution to the deformation of a minimal-energy state arises from core polarisation of the nucleus by the quadrupole field of the SU(3) states, the minimal-energy state should be one for which the polarisation is maximally aligned with the SU(3) deformation. Inspection of Fig. 9 shows that such an alignment is achieved when the rotational angle $\Omega^{\prime}$ is zero. It then remains to determine the scale deformation for which $E(\mathbf{d})$ is a minimum.

We start from the following observations:

(i) By construction, the lowest-weight state $|\sigma\rangle=\left|N_{0}\left(\lambda_{0} \mu_{0}\right)\right\rangle$ is an eigenstate of an isotropic harmonic-oscillator Hamiltonian

$$
\hat{H}_{0}=\sum_{n}\left[\frac{\hat{\mathbf{p}}_{n}^{2}}{2 M}+\frac{1}{2} M \omega^{2}\left(x_{n 1}^{2}+x_{n 2}^{2}+x_{n 3}^{2}\right)\right]
$$


with eigenvalue given by

$$
\hat{H}_{0}|\sigma\rangle=N_{0} \hbar \omega|\sigma\rangle .
$$

(ii) Every state $|\sigma(g)\rangle \in \mathscr{R}$ is a lowest-weight state for an $\operatorname{Sp}(3, \mathbb{R})$ irrep with respect to some harmonic-oscillator Hamiltonian.

(iii) The lowest-weight state $|\sigma(\mathbf{d})\rangle$ is an eigenstate of a triaxial harmonic oscillator

$$
\hat{H}_{\mathbf{d}}=\sum_{n}\left[\frac{\hat{\mathbf{p}}_{n}^{2}}{2 M}+\frac{1}{2} M\left(\omega_{1}^{2} x_{n 1}^{2}+\omega_{2}^{2} x_{n 2}^{2}+\omega_{3}^{2} x_{n 3}^{2}\right)\right] .
$$

We now determine the frequencies $\left\{\omega_{i}\right\}$ of this triaxial oscillator for the minimalenergy state by use of the self-consistency property of mean-field theory which is that the minimum-energy state should be one for which (to the extent allowed by the mean-field constraints) the equidensity surfaces of the state have the same shape as the corresponding equipotential surfaces of the independent-particle Hamiltonian of which it is an eigenstate.

It is important to understand that, although the lowest weight $|\sigma\rangle$ is observed to be an eigenstate of an independent-particle Hamiltonian, there is no implication that $|\sigma\rangle$ is an independent-particle state. However, in many important cases, e.g., for an $\operatorname{Sp}(3, \mathbb{R})$ irrep built on a leading SU(3) irrep, it may be.

An ellipsoidal surface associated with the density distribution of the lowest-weight state $|\sigma\rangle$ is defined by the expectation values

$$
\left\langle x_{i}^{2}\right\rangle_{\sigma}=\left\langle\sigma\left|\sum_{n} x_{n i}^{2}\right| \sigma\right\rangle=\frac{\hbar N_{i}}{M \omega}, \quad i=1,2,3,
$$

and, unlike the isotropic harmonic-oscillator Hamiltonian (48) for which this state is an eigenstate, it is not spherically symmetric. On the other hand, a state $|\sigma(d)\rangle$ which is an eigenstate of an anisotropic harmonic oscillator with potential

$$
V(x)=\frac{1}{2} M \sum_{n}\left(\omega_{1}^{2} x_{n 1}^{2}+\omega_{2}^{2} x_{n 2}^{2}+\omega_{3}^{2} x_{n 3}^{2}\right),
$$

has the mean values

$$
\left\langle x_{i}^{2}\right\rangle_{\sigma(\mathbf{d})}=\left\langle\sigma(\mathbf{d})\left|\sum_{n} x_{n i}^{2}\right| \sigma(\mathbf{d})\right\rangle=\frac{\hbar N_{i}}{M \omega_{i}},
$$

An ellipsoidal equi-density surface for this state, defined by the equation

$$
\frac{x_{1}^{2}}{\left\langle x_{1}^{2}\right\rangle_{\sigma(\mathbf{d})}}+\frac{x_{2}^{2}}{\left\langle x_{2}^{2}\right\rangle_{\sigma(\mathbf{d})}}+\frac{x_{3}^{2}}{\left\langle x_{3}^{2}\right\rangle_{\sigma(\mathbf{d})}}=\text { const. }
$$

is then given by

$$
\frac{\omega_{1} x_{1}^{2}}{N_{1}}+\frac{\omega_{2} x_{2}^{2}}{N_{2}}+\frac{\omega_{3} x_{3}^{2}}{N_{3}}=1
$$


whereas an equi-potential surface for the potential (52) is given by

$$
\omega_{1}^{2} x_{1}^{2}+\omega_{2}^{2} x_{2}^{2}+\omega_{3}^{2} x_{3}^{2}=1
$$

Thus, for these surfaces to have the same shape, it is required that

$$
N_{1} \omega_{1}=N_{2} \omega_{2}=N_{3} \omega_{3}=k
$$

for some value of $k$.

The volume of the ellipsoid (55) is

$$
\text { vol. }=\frac{4 \pi}{3}\left(\frac{N_{1} N_{2} N_{3}}{\omega_{1} \omega_{2} \omega_{3}}\right)^{\frac{1}{2}} .
$$

Thus, in order that this volume should be the same as that for $\omega_{1}=\omega_{2}=\omega_{3}=\omega$, we set

$$
\omega_{1} \omega_{3} \omega_{3}=\omega^{3}
$$

and obtain

$$
k^{3}=N_{1} N_{2} N_{3} \omega^{3} .
$$

This means that the scale parameters for the minimal-energy state should be such that

$$
\frac{\left\langle x_{i}^{2}\right\rangle_{\sigma(d)}}{\left\langle x_{i}^{2}\right\rangle_{\sigma}}=\frac{\omega}{\omega_{i}}=\frac{N_{i} \omega}{k}=\frac{N_{i}}{\left(N_{1} N_{2} N_{3}\right)^{1 / 3}} .
$$

The above self-consistency method extends to all orders the methods used in Refs. $[25,26,27]$ to estimate the strength $\chi=\hbar \omega / N_{0}$ to leading order in $\lambda / N_{0}$ and $\mu / N_{0}$, of the coupling constant for the effective quadrupole-quadrupole interaction in a model Hamiltonian of the form $\hat{H}=\hat{H}_{\mathrm{sp}}-\frac{1}{2} \chi Q \cdot Q$.

Several important consequences follow from this shape-consistency result.

(i) Coupling to giant resonance states (essentially) doubles the values of SU(3) quadrupole moments.

This follows because quadrupole moments of the state $|\sigma\rangle$ are given in the SU(3) model in a lowest-grade irrep, in terms of the oscillator unit $a=\sqrt{M \omega / \hbar}$, by

$$
\begin{aligned}
& a^{2}\left\langle\left(2 x_{1}^{2}-x_{2}^{2}-x_{3}^{2}\right)\right\rangle_{\sigma}=2 N_{1}-N_{2}-N_{3}=2 \lambda_{0}+\mu_{0}, \\
& a^{2} \sqrt{\frac{2}{3}}\left\langle\left(x_{2}^{2}-x_{3}^{2}\right)\right\rangle_{\sigma}=N_{2}-N_{3}=\mu_{0},
\end{aligned}
$$

Therefore, for the shape-consistent state $|\sigma(\mathbf{d})\rangle$, they are given by

$$
\begin{aligned}
& a^{2}\left\langle 2 x_{1}^{2}-x_{2}^{2}-x_{3}^{2}\right\rangle_{\sigma(\mathbf{d})}=\frac{2 N_{1}^{2}-N_{2}^{2}-N_{3}^{2}}{\left(N_{1} N_{2} N_{3}\right)^{1 / 3}}, \\
& a^{2} \sqrt{\frac{2}{3}}\left\langle x_{2}^{2}-x_{3}^{2}\right\rangle_{\sigma(\mathbf{d})}=\frac{N_{2}^{2}-N_{3}^{2}}{\left(N_{1} N_{2} N_{3}\right)^{1 / 3}} .
\end{aligned}
$$


An expansion in powers of $\lambda_{0} / N_{0}$ and $\mu_{0} / N_{0}$ then gives

$$
\begin{aligned}
& a^{2}\left\langle 2 x_{1}^{2}-x_{2}^{2}-x_{3}^{2}\right\rangle_{\sigma(\mathbf{d})}=2\left(2 \lambda_{0}+\mu_{0}\right)+\left(2 \lambda_{0}^{2}+2 \lambda_{0} \mu_{0}-\mu_{0}^{2}\right) / N_{0}+\ldots, \\
& a^{2} \sqrt{\frac{2}{3}}\left\langle x_{2}^{2}-x_{3}^{2}\right\rangle_{\sigma(\mathbf{d})}=2 \mu_{0}-\left(2 \lambda_{0} \mu_{0}-\mu_{0}^{2}\right) / N_{0}+\ldots
\end{aligned}
$$

which, to leading order in $\lambda_{0} / N_{0}$ and $\mu_{0} / N_{0}$, imply that the effective charge of quadrupole moments and E2 transitions in the SU(3) model is predicted to be

$$
e_{\text {eff }}=(2+\text { higher order terms. }) \times \text { the bare nucleon charge, }
$$

where the bare nucleon charge for an SU(3) irrep would normally be $Z / A$ times the proton charge.

(ii) Coupling to the giant resonance excitations lowers the energies of deformed states. To estimate the magnitude of this lowering, one should compare the expectation values of $\langle\sigma|\hat{H}| \sigma\rangle$ and $\langle\sigma(\mathbf{d})|\hat{H}| \sigma(\mathbf{d})\rangle$ for a realistic nuclear Hamiltonian. However, in the spirit of the shell model in which a first energy-ordering of states is given by their harmonic oscillator energies, we consider the energy of the state $|\sigma(d)\rangle$ for the shape-consistent harmonic oscillator Hamiltonian $\hat{H}_{d}$ of Eq. (50). This gives

$$
E_{N_{1}, N_{2}, N_{3}}=\left\langle\sigma(\mathbf{d})\left|\hat{H}_{\mathbf{d}}\right| \sigma(\mathbf{d})\right\rangle=3 \hbar k=3\left(N_{1} N_{2} N_{3}\right)^{1 / 3} \hbar \omega,
$$

which is less than the energy of the lowest-weight state for the same irrep relative to the spherical harmonic oscillator Hamiltonian

$$
E_{N_{0}}=N_{0} \hbar \omega=\left(N_{1}+N_{2}+N_{3}\right) \hbar \omega .
$$

A consequence of this result is:

(iii) The lowest-energy shell-model states of well-deformed nuclei are unlikely to lie in the lowest-energy spherical-harmonic oscillator shells.

This claim is based on the observation that an $\operatorname{Sp}(3, \mathrm{R})$ irrep is defined uniquely by the quantum numbers $N_{1}, N_{2}$, and $N_{3}$ for which

$$
N_{0}=N_{1}+N_{2}+N_{3}, \quad \lambda_{0}=N_{1}-N_{2}, \quad \mu_{0}=N_{2}=N_{3} .
$$

Moreover, all the mean-field states $\{|\sigma(g)\rangle, g \in \operatorname{Sp}(3, \mathrm{R})\}$ for a given $\operatorname{Sp}(3, \mathrm{R})$ irrep have common values for these quantum numbers and are of lowest-weight for the $\operatorname{Sp}(3, \mathrm{R})$ irrep with respect to some (generally triaxial) harmonic oscillator Hamiltonian. We have argued that, with respect to any reasonable nuclear Hamiltonian, the lowest energy mean-field state should be one for which there is shape consistency between the density of the minimal-energy state and the harmonic oscillator Hamiltonian for which the minimal-energy state is an eigenstate. A property of standard Hartree-Fock theory, on manifolds of Slater determinants, is that the minimal-energy state with respect to a given nuclear Hamiltonian is also of minimal energy with respect to the locally defined Hartree-Fock independent-particle 
Hamiltonian. If we assume this property to hold for the above described $\operatorname{Sp}(3, \mathrm{R})$ mean-field theory, for which the analogue of the Hartree-Fock independent-particle Hamiltonian is the anisotropic harmonic oscillator Hamiltonian for which a given lowest-weight $\operatorname{Sp}(3, \mathrm{R})$ state is an eigenstate, the minimal energy state with respect to the given nuclear Hamiltonian should also be a minimal energy state with respect to the locally-defined harmonic-oscillator Hamiltonian.

As noted above, the lowest-weight state $|\sigma\rangle$ of an $\operatorname{Sp}(3, \mathrm{R})$ irrep $\left\langle N_{0}\left(\lambda_{0} \mu_{0}\right)\right\rangle$ with respect to an isotropic harmonic oscillator Hamiltonian is an eigenstate of this Hamiltonian with energy $N_{0} \hbar \omega$. However, the shape-consistent lowest-weight state $|\sigma(d)\rangle$ of the same volume is an eigenstate of an anisotropic harmonic oscillator of energy eigenvalue $E_{N_{1}, N_{2}, N_{3}}=3\left(N_{1} N_{2} N_{3}\right)^{1 / 3} \hbar \omega$, which is less than $N_{0} \hbar \omega$ unless $N_{1}, N_{2}$, and $N_{3}$ are all equal. Thus, it is appropriate to compare the minimal energies $E_{N_{1}, N_{2}, N_{3}}$ for the possible $\operatorname{Sp}(3, \mathrm{R})$ irreps that are available to a given nucleus.

\subsection{Identifying the lowest-energy $\operatorname{Sp}(3, R)$ irreps}

The above shape-consistent mean-field results suggest that an energy ordering of the $\operatorname{Sp}(3, \mathrm{R})$ irreps available to a given nucleus is given by the values of $E_{N_{1}, N_{2}, N_{3}}$. For the given nucleus, we can list the possible values of $N_{1}, N_{2}$, and $N_{3}$ together with the corresponding values of $E_{N_{1}, N_{2}, N_{3}}$. The first few irreps in order of increasing $E_{N_{1}, N_{2}, N_{3}}$ are shown for ${ }^{12} \mathrm{C},{ }^{16} \mathrm{O}$ and ${ }^{168} \mathrm{Er}$ in Table 1.

TABLE 1. Minimal mean-field energies $E_{N_{1} N_{2} N_{3}}$ in units of $\hbar \omega$ for the lowest-energy irreps of ${ }^{12} \mathrm{C},{ }^{16} \mathrm{O}$, and ${ }^{168} \mathrm{Er}$ as defined in the text.

\begin{tabular}{|c|c|c|c|}
\multicolumn{4}{|c|}{${ }^{12} \mathrm{C}$} \\
\hline$N_{0}$ & $\lambda_{0}$ & $\mu_{0}$ & $E_{N_{1} N_{2} N_{3}}$ \\
\hline 26 & 0 & 4 & 25.3 \\
30 & 12 & 0 & 26.0 \\
28 & 6 & 2 & 26.3 \\
32 & 10 & 2 & 28.6 \\
\hline
\end{tabular}

\begin{tabular}{|c|c|c|c|}
\multicolumn{4}{|c|}{${ }^{16} \mathrm{O}$} \\
\hline$N_{0}$ & $\lambda$ & $\mu$ & $E_{N_{1} N_{2} N_{3}}$ \\
\hline 36 & 0 & 0 & 36 \\
40 & 8 & 4 & 37.3 \\
38 & 4 & 2 & 37.3 \\
40 & 7 & 3 & 38.1 \\
48 & 24 & 0 & 38.1 \\
44 & 16 & 2 & 38.3 \\
\hline
\end{tabular}

\begin{tabular}{|c|c|c|c|}
\multicolumn{4}{|c|}{${ }^{168} \mathrm{Er}$} \\
\hline$N_{0}$ & $\lambda$ & $\mu$ & $E_{N_{1} N_{2} N_{3}}$ \\
\hline 814 & 30 & 8 & 812.6 \\
826 & 96 & 20 & 812.9 \\
822 & 70 & 28 & 813.0 \\
818 & 52 & 20 & 813.1 \\
816 & 42 & 12 & 813.1 \\
830 & 114 & 16 & 813.2 \\
\hline
\end{tabular}

The first remarkable result is that for the light nucleus ${ }^{12} \mathrm{C}$ the lowest three $\operatorname{Sp}(3, \mathrm{R})$ irreps are consistent with the states observed in this nucleus as described in an $\operatorname{Sp}(3, \mathrm{R})$ model framework by Dreyfuss et al. [28, 29] whose results are shown in Fig. 10. In particular, the highly deformed second excited state, known as the Hoyle state, which belongs to the $\left(\lambda_{0}, \mu_{0}\right)=(120)$ irrep, falls well below its unperturbed energy of $4 \hbar \omega$ in the spherical harmonic oscillator shell model. A similar, result is found for ${ }^{16} \mathrm{O}$, for which the lowest three irreps are consistent with those observed and described [27] in an $\mathrm{Sp}(3, \mathrm{R})$ model framework in Fig. 11. For ${ }^{16} \mathrm{O}$, the first-excited state belongs to an irrep whose lowest-weight state would again be $4 \hbar \omega$ above its unperturbed spherical harmonic oscillator and would lie outside of any normal shell-model valence space. 


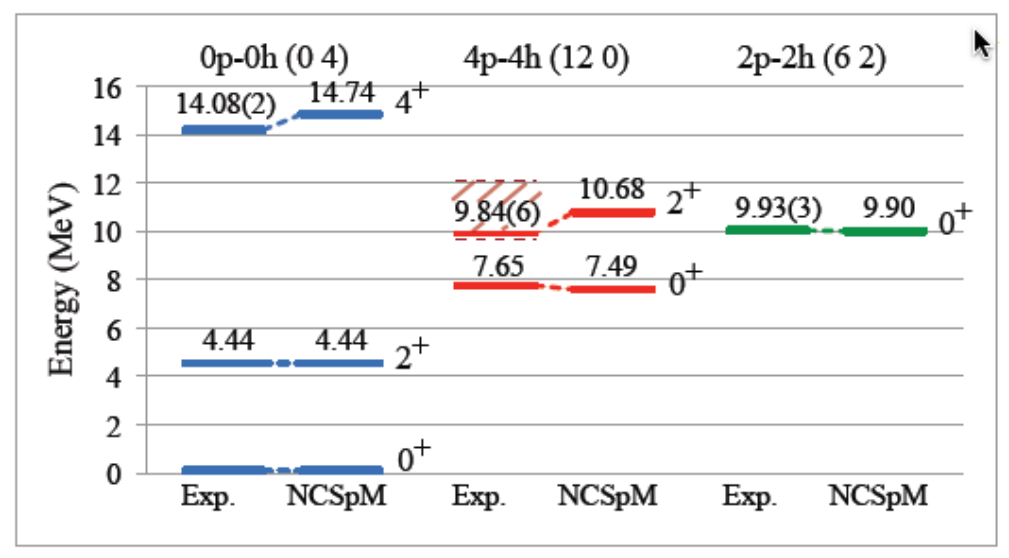

FIGURE 10. The low-energy spectrum of ${ }^{12} \mathrm{C}$ energies calculated for the first three $\mathrm{Sp}(3, \mathrm{R})$ irreps shown for ${ }^{12} \mathrm{C}$ in Table 1 by Dreyfuss et al. [29]. (The figure is from their preprint with permission.)

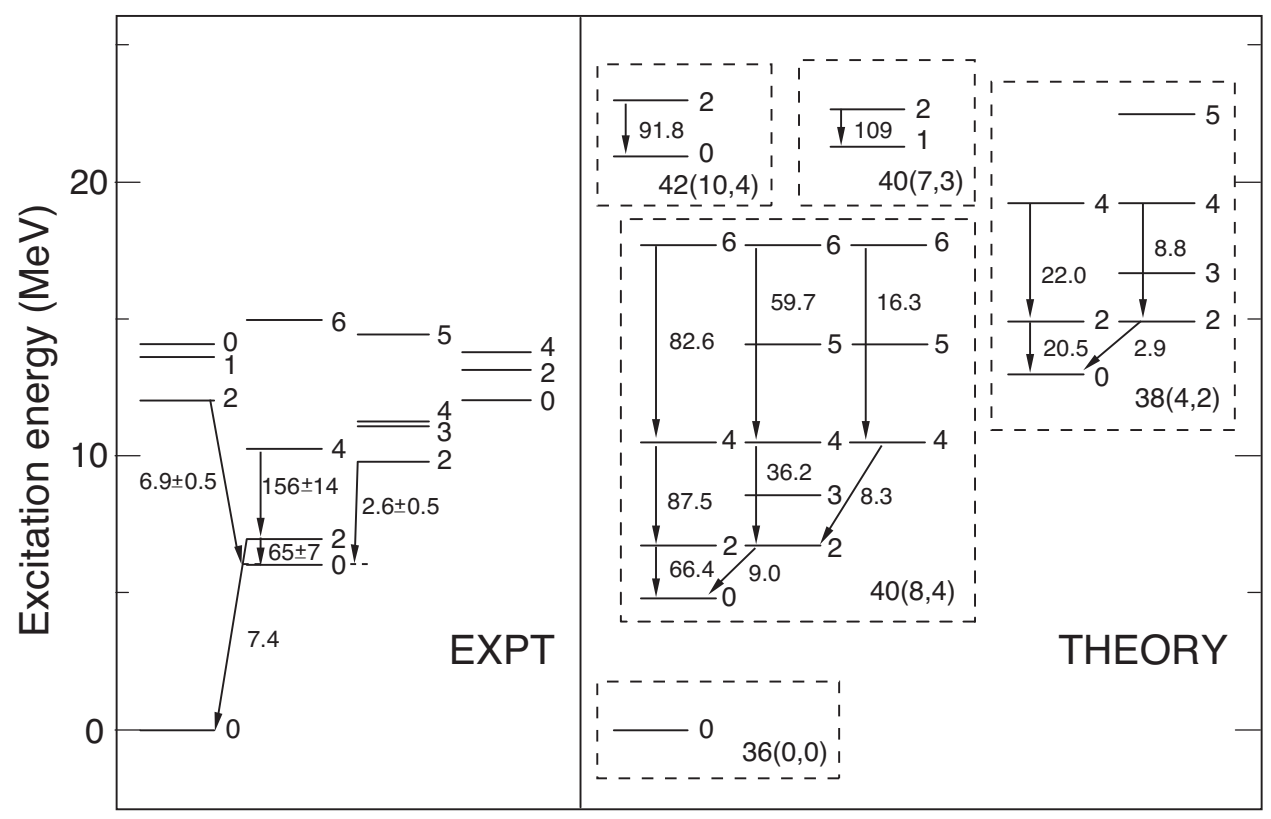

FIGURE 11. The low-energy spectrum of ${ }^{16} \mathrm{O}$ energies calculated for a few $\mathrm{Sp}(3, \mathrm{R})$ irreps. (The figure is from Ref. [27].)

For a range of Erbium and Tungsten isotopes, Jarrio et al. [24] estimated the most appropriate $\operatorname{Sp}(3, \mathrm{R})$ irreps needed to describe the ground-state rotational bands of these nuclei directly from experiments. Their method was simply to fit the E2 transition-rate data with an $\operatorname{Sp}(3, \mathrm{R})$ irrep in its rotor-model limit. They determined, for example, that the mean values of $\lambda_{0}$ and $\mu_{0}$, for the low-energy states of ${ }^{168} \mathrm{Er}$ are approximately $\left\langle\lambda_{0}\right\rangle \approx 80$ and $\left\langle\mu_{0}\right\rangle \approx 12$. They also determined the mean values of these quantum numbers for Nilsson model states at the experimentally determined deformation and obtained the values $\left\langle\lambda_{0}\right\rangle \approx 95$ and $\left\langle\mu_{0}\right\rangle \approx 12$. Given that there is undoubtedly a mixing of $\operatorname{Sp}(3, \mathrm{R})$ irreps due to symmetry breaking interactions, their results are reasonably consistent with 
the dominant $\operatorname{Sp}(3, \mathrm{R})$ irrep contributing to the ground-state band of ${ }^{168} \mathrm{Er}$ being the irrep with the next to lowest $E_{N_{1} N_{2} N_{3}}$ energy given for ${ }^{168} \mathrm{Er}$ in Table 1 . But they are inconsistent with the much less deformed irrep with $N_{0}\left(\lambda_{0} \mu_{0}\right)=814(308)$ and a slightly lower value of $E_{N_{1} N_{2} N_{3}}$, which is the most deformed irrep that could be obtained in the standard spherical shell model. What is especially notable is that to obtain an $\operatorname{Sp}(3, R)$ irrep, with the large experimentally-observed deformation, it is necessary to go to unperturbed spherical-harmonic-oscillator shell-model states of $12 \hbar \omega$ above the lowest-energy irrep of $N_{0}=814$.

\subsection{The wave functions of a symplectic model calculation}

Several algebraic symplectic model calculations have been made, where by algebraic we mean calculations with phenomenological Hamiltonians that are low-order polynomials in the $\operatorname{Sp}(3, \mathbb{R})$ Lie algebra. Such calculations, which explore the physical content of the symplectic model and its idiosynchracies in preparation for more detailed microscopic studies, became relatively straightforward following the development of vector coherent state methods $[30,31,32]$ for calculating the matrix elements of the $\operatorname{Sp}(3, R)$ Lie algebra.

Results of a calculation [23] of the energy levels of the ground-state rotational band of ${ }^{166} \mathrm{Er}$ are shown in Fig. 8. The corresponding wave functions, shown in Fig. 12, are particularly revealing. They are very different from those calculated both with algebraic

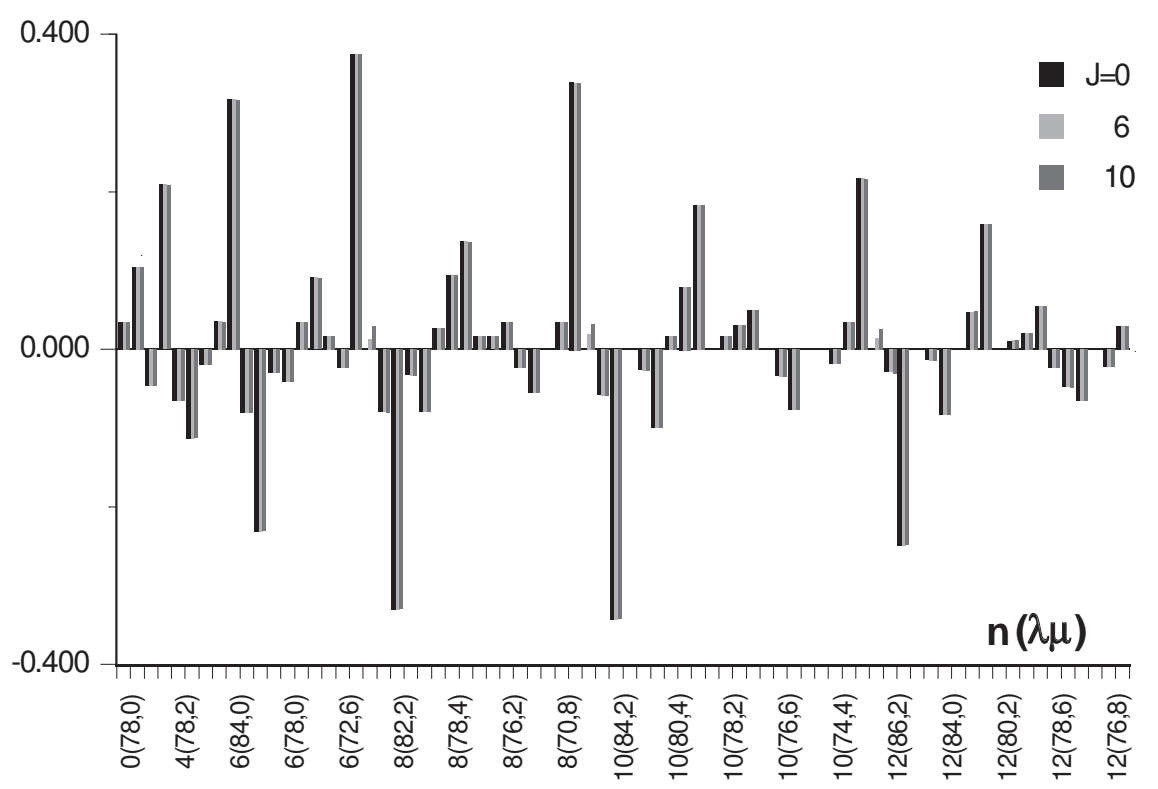

FIGURE 12. Amplitudes for the wave functions of the $J=0,6$, and $10, \operatorname{Sp}(3 . \mathrm{R})$ model states of figure 8 in a $\mathrm{U}(3)$ basis. The distribution of basis states includes states from 6 major harmonic-oscillator shells of positive parity that span a spherical harmonic-oscillator energy range of $12 \hbar \omega$. Note that the SU(3) amplitudes of all states are essentially independent of their angular momenta $J$. 
and microscopic interactions for light nuclei ${ }^{1}$ which tend to have their dominant components in the lowest spherical harmonic-oscillator shells and contributions from higher shells that fall off rapidly over the space of a few shells. In contrast, the amplitude of the lowest-grade U(3) irrep, 826.5(78 0), labelled 0(78 0) in the figure, which is the left-most amplitude shown in the figure, is seen to represent a minuscule component of the states of the rotational band calculated. Given that the lowest-grade U(3) irrep $826.5(780)$ is already at an excitation of $12 \hbar \omega$ relative to a spherical harmonic oscillator shell-model Hamiltonian, the amplitudes shown in this figure are a clear indication of the irrelevance of shell-model calculations carried out in conventional shell-model spaces. However, from a consideration of Nilsson model states for rotational bands in highly-deformed nuclei, this comes as no surprise.

\subsection{Some conclusions}

The above results lead to the following conclusions:

(i) The partial ordering of $\operatorname{Sp}(3, R)$ irreps by the spherical harmonic oscillator energies of their lowest-weight states, $N_{0} \hbar \omega$, is inappropriate for deformed nuclei.

Recall that spin-orbit interactions also brings about a significant re-arrangment of the single-particle energy levels of the spherical harmonic oscillator and that pseudo-SU(3) [34] and pseudo-symplectic models [35] have been proposed to take this into account. The above considerations suggest that the re-ordering of the relevant shell-model configurations due to deformation is an even larger concern in strongly-deformed nuclei. Even so, the mixing of symplectic irreps by spin-orbit and other interactions, notably pairing interactions, has to be considered and is addressed briefly in the following section. Thus, the potentiality for combining the pseudo-symplectic techniques and the above methods is worth considering.

(ii) The symplectic model in combination with self-consistent mean-field considerations demonstrates the importance of the quadrupole component of the mean field and leads to the expectation of coexisting states of different deformation already in the low-energy domain.

The best known examples of this in light nuclei are the Hoyle state at $7.65 \mathrm{MeV}$ in ${ }^{12} \mathrm{C}$ and the first excited, 4-particle-4 hole, state in ${ }^{16} \mathrm{O}$, Many other examples of shape coexistence are given in the recent review of Heyde and Wood [22].

(iii) The dominance of highly deformed states in large open-shell nuclei, such as those of the rare-earth region, is an indication that, while the self-consistency formula, which orders $\operatorname{Sp}(3, R)$ irreps by $E_{N_{1} N_{2} N_{3}}$, provides a first estimate of the lowering of deformed states in heavy nuclei, it is probably an underestimate.

It would appear to be an observed fact that highly-deformed irreps fall below the lowest-energy states of the spherical shell model in large doubly-open-shell nuclei. However, it unclear how far below they fall. This suggests the importance

\footnotetext{
${ }^{1}$ See the several results reviewed in Ref. [33].
} 
of seeking evidence of spherical excited states in otherwise deformed nuclei, which might correspond to closed sub-shell configurations (see [22]). It also suggests the importance of obtaining more reliable results from mean-field calculations in the spaces of $\operatorname{Sp}(3, \mathrm{R})$ irreps with meaningful nucleon-nucleon interactions.

\section{MICROSCOPIC CALCULATIONS IN SP(3, R) BASES}

The concern of this section is with the use of the subgroup chain

$$
\operatorname{Sp}(3, \mathrm{R}) \supset \mathrm{U}(3) \supset \mathrm{SO}(3) \text {, }
$$

to define shell-model coupling schemes for microscopic calculations. For a complete scheme, the basis states of this chain are combined with complementary spin-isospin states classified by the supermultiplet chain

$$
\mathrm{U}(4) \supset \mathrm{SO}(4) \supset \mathrm{SU}(2)_{S} \times \mathrm{SU}(2)_{T}
$$

in an isospin formalism, or their neutron-proton counterparts,

Enormous progress has been made by Dytrych et al. [36, 37] in the development of efficient shell-model calculations in $\mathrm{Sp}(3, \mathrm{R}) \supset \mathrm{SU}(3)$ bases and more general multishell SU(3) bases for no-core shell model calculations. These developments are of fundamental importance for showing that the many-nucleon theory of nuclear physics can be derived with nucleon interactions obtained from experiment, meson theory, and effective field theory (see lectures of Machleidt).

It is to be hoped that many of the essential techniques developed in $[36,37]$ will also apply to heavy nuclei. The problem is the explosion of the dimensions of the nocore shell model approach with the number of spherical harmonic oscillator shells in the active space of the calculation, which Fig. 12, for example, shows can be be very large for heavy deformed nuclei. Moreover, as this overview indicates, they are expected to lie well above the lowest-energy harmonic-oscillator shells. Thus, we explore a complementary approach for applications to well-deformed states in rotational nuclei which reduces the dimensions of the $\operatorname{Sp}(3, \mathrm{R})$ spaces needed for converged results by several orders of magnitude.

\subsection{Calculations within a single $\operatorname{Sp}(3, R)$ irrep}

We consider a generator coordinate approach based on a procedure proposed by Filippov, Okhrimenko, Vasilevsky, Vassanji, and others [38, 39, 40, 41] and further developed by Carvalho, and Rowe [42, 43, 44].

This approach is founded on the observation that the real submanifold $\mathscr{R}$ of $\operatorname{Sp}(3, \mathrm{R})$ coherent states (44) spans the Hilbert space of the corresponding $\operatorname{Sp}(3, \mathrm{R})$ irrep. Thus, a basis for a truncated subspace is given by a discrete set of states

$$
\left|N_{0}\left(\lambda_{0} \mu_{0}\right) i j k\right\rangle=\hat{R}\left(\Omega_{i}\right) \hat{U}\left(\mathbf{d}_{j}\right) \hat{R}\left(\Omega_{k}^{\prime}\right)\left|N_{0}\left(\lambda_{0} \mu_{0}\right)\right\rangle,
$$


where $\left|N_{0}\left(\lambda_{0} \mu_{0}\right)\right\rangle$ is the lowest-weight state for the irrep. The notable fact, shown in Ref. [44], is that converged results are obtained using this method, to a given level of accuracy, with very few points when chosen in an optimal way.

Good choices can be made following a computation of the mean field-energy $E\left(\Omega \mathrm{d} \Omega^{\prime}\right)$ defined by Eq. (47). For a rotationally invariant Hamiltonian, this energy is independent of $\Omega$, i.e., $E\left(\Omega \mathrm{d} \Omega^{\prime}\right)=E\left(\mathrm{~d} \Omega^{\prime}\right)$. Taking linear combinations of states of many $\Omega_{i}$ then amounts to angular-momentum projection and the generation of states with good angular-momentum quantum numbers for which techniques have been developed, for example, by Cusson and Lee [45]. With only a single diagonal matrix $\overline{\mathbf{d}}$, namely that for which $E(\overline{\mathbf{d}})$ is a minimum and only the null rotation for $\Omega^{\prime} \in \operatorname{SO}(3)$, it is found that rather good results are already obtained. However, the results, for a fixed value of $\mathbf{d}$ do not allow for centrifugal stretching effects corresponding to changes in the mean values of the elements of $\mathbf{d}$ with increasing angular momentum. Such effects are included by allowing $\mathbf{d}$ to vary with the angular momentum or by taking a small fixed set of $\left\{\mathbf{d}_{j}\right\}$ about the mean-field minimum value $\overline{\mathbf{d}}$. (The preponderance of experimental evidence, based on yrast B(E2) values, suggests nearly constant $d$ values.) Optimal $\left\{\mathbf{d}_{j}\right\}$ sets can be determined along the lines considered by Carvalho et al. [44] in consideration of the energy functions $E(\mathbf{d})$ and the range of values of $\mathbf{d}$ about the optimal value $\overline{\mathbf{d}}$ for which $\left\langle N_{0}\left(\lambda_{0} \mu_{0}\right)\left|U^{\dagger}(\mathbf{d}) U(\overline{\mathbf{d}})\right| N_{0}\left(\lambda_{0} \mu_{0}\right)\right\rangle$ is non-negligible. Similar choices can be made for $\left\{\Omega_{k}^{\prime}\right\}$.

Applications of this approach to date obtain promising results with just a few $\left\{\mathbf{d}_{j}\right\}$ and neglect of the $\Omega^{\prime}$ degree of freedom. Neglect of the $\Omega^{\prime}$ degree of freedom has an interesting physical significance. Note that an $N_{0}\left(\begin{array}{ll}0 & 0\end{array}\right)$ irrep, in which $\Omega^{\prime}$ does not contribute, contains the spectrum of $\mathrm{U}(3)$ irreps

$$
\left\{\left\{N_{0}+n\left(n_{1}-n_{2}, n_{2}-n_{3}\right)\right\}, n=0,2,4, \ldots\right\}
$$

where $n_{1}, n_{2}$, and $n_{3}$ are even integers and $n_{1} \geq n_{2} \geq n_{3} \geq 0$. Thus, as illustrated in Fig, 5 , the $\mathrm{U}(3)$ spectrum of states for a generic $\operatorname{Sp}(3, \mathrm{R})\left\langle N_{0}\left(\lambda_{0} \mu_{0}\right)\right\rangle$ is given by the tensor products of the SU(3) irrep $\left(\lambda_{0} \mu_{0}\right)$ with the U(3) irreps in the set (75). With suppression of the $\Omega^{\prime}$ degree of freedom, some of the irreps in this tensor product will be missing. However, no irrep of the stretched product form

$$
\left\{N_{0}+n\left(\lambda_{0}+n_{1}-n_{2}, \mu_{0}+n_{2}-n_{3}\right)\right\}, \quad n=0,2,4, \ldots
$$

is omitted. But, which precisely which irreps are missing is not obvious to me at this time. This needs investigation and, if necessary, the $\Omega^{\prime}$ degree of freedom can be included.

The technology for the efficient application of the above generator coordinate procedure remains to be fully developed. So far, it has been applied to $\operatorname{Sp}(3, \mathbb{R})$ irreps of maximal space symmetry; these are spaces of zero neutron and proton intrinsic spins. More generally, it is necessary to consider an irrep of the direct-product group $\mathrm{Sp}(3, \mathrm{R}) \times \mathrm{SU}(4)$, in an isospin formalism, or the tensor product of two irreps of the direct product $\mathrm{Sp}(3, \mathrm{R}) \times \mathrm{SU}(2)$, in a neutron-proton formalism. 


\subsection{Mixed-representation calculations in an $\operatorname{Sp}(3, R) \supset U(3)$ basis and quasi-dynamical symmetry}

The development of efficient programs for the calculation of states of even-even nuclei within spaces of multiple $\operatorname{Sp}(3, \mathrm{R})$ irreps of maximum space symmetry (i.e., spaces of zero neutron and proton intrinsic spin) will undoubtedly be achieved in the near future. Exploratory calculations within such spaces are needed, starting with model interactions, to identify the nature of any problems that might arise, before the bigger task of elaborating apposite techniques for mixed representations with non-zero intrinsic spins and microscopic interactions is tackled. This task would appear to be challenging but not insurmountable. Given that a relatively small number of optimally-chosen generatorcoordinate basis states is needed, for each $\operatorname{Sp}(3, \mathbb{R})$ irrep, to obtain an acceptable level of converged solutions, and because rotational structure is much more clearly defined in heavy nuciei, it is possible that microscopic calculations in heavy deformed nuclei will eventually prove to be little, if any, more difficult than for the less deformed states of light nuclei.

Calculations in spaces of mixed $\operatorname{Sp}(3, \mathrm{R})$ irreps are necessary to understand the influences on rotational structures arising from various $\operatorname{Sp}(3, \mathrm{R})$ symmetry-breaking interactions, such as spin-orbit interactions and pairing interactions. Some years ago there was concern [46] that, while systems with a dynamical symmetry can be handled with relative ease, systems with two or more competing dynamical symmetries would generally be intractable. However, it was also known that, if the dynamics associated with one of a competing pair of dynamical symmetries was slow and the other fast, there could be an adiabatic separation of the variables along the lines proposed by Born and Oppenheimer [47]. This concept was therefore incorporated into the language of group theory in terms of the precisely-defined mathematical concept of an embedded representation [48]. Such representations were subsequently observed to arise approximately in many situations of competing dynamical symmetries and were described as quasi-dynamical symmetries [49].

For a review of systems with competing dynamical symmetries see [50]. Such systems commonly exhibit a quantum phase transition between a phase in which one dynamical symmetry is dominant and another phase in which a competing dynamical symmetry is dominant. Moreover, the transition between the two phases typically becomes sharper for a many-particle system as the particle number is increased. It appears that such a dominant symmetry is almost invariably characteristic of a quasi-dynamical symmetry.

The prototype of a quasi-dynamical symmetry is given by a system that has the dynamical symmetry of a rigid rotor with irreps defined by fixed intrinsic values of its quadrupole moments. When the rigid-rotor irreps are mixed by dynamical symmetrybreaking interactions, the system becomes a soft rotor with a distribution of intrinsic quadrupole moments. However, in the adiabatic limit, in which the centrifugal and Coriolis forces are weak, the intrinsic distribution remains constant over a range of lowenergy angular-momentum states of its ground-state rotational band. In isolation, these states then have the same relative properties as those of a rigid-rotor; their non-rigid intrinsic structures are only revealed by generally small but non-zero transition matrix elements to states of excited bands. 
The following example [51] of an $N=48$ particle system with a Hamiltonian, given as function of a control parameter $\alpha$ by

$$
\hat{H}(\alpha)=\hat{H}_{0}+(1-\alpha) \hat{H}_{\mathrm{SU} 2}+\alpha \hat{H}_{\mathrm{SU} 3},
$$

illustrates a close approach to a sharp phase transition from one phase to another as $\alpha$ is increased past a critical point. The low-energy spectrum of this Hamiltonian is shown, as a function of $\alpha$ in Fig. 13. When $\alpha=0$ the Hamiltonian has an SU(2) $\subset$ USp(6) dynamical symmetry and the system is in a superconducting vibrational phase. And, when $\alpha=1$ the Hamiltonian has an SU(3) $\subset \mathrm{USp}(6)$ dynamical symmetry and the system is in an adiabatic SU(3)-rotational phase (with low excitation energies). For intermediate values of $\alpha$ the two dynamical symmetries are in competition. The remarkable fact is that the spectrum is characteristic of the SU(2) phase for $\alpha \lesssim 0.55$ and characteristic of the SU(3) phase for $\alpha \gtrsim 0.60$. Moreover, as the particle number is increased the transition point becomes increasingly sharp.

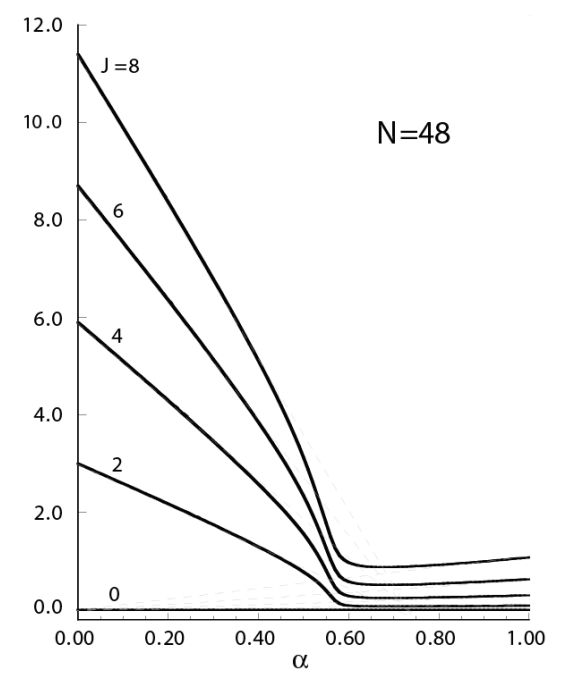

FIGURE 13. The low-energy spectrum for the model Hamiltonian (77) as a function of $\alpha$ for a system of $N=48$ particles.

The corresponding wave functions for three values of $\alpha$ are shown in Fig. 14. What is notable is the sudden change in the character of the wave functions as $\alpha$ increases past its critical value of $\approx 0.58$ As $\alpha$ increases above the critical point, the SU(3) amplitudes show a reduced mixing of irreps but, more significantly, the mixed SU(3) structure of each state of the quasi-SU(3) rotational band rapidly becomes the same for each of the states in the band. It is as if all the SU(3) irreps were equivalent. It appears that only $\mathrm{SU}(3)$ irreps with similar quantum numbers are mixed strongly by symmetry-breaking interactions.

The reduction in the mixing of basis states belonging to widely different irreps of a dominant dynamical symmetry is illustrated and can be understood in the ${ }^{12} \mathrm{C}$ and ${ }^{16} \mathrm{O}$ calculations mentioned above. For these nuclei, the mixing of the spherical harmonic oscillator lowest-energy configurations with those that form the highly deformed firstexcited states is much suppressed by the fact that all matrix elements of one- and twobody operators between the low-lying $\operatorname{Sp}(3, \mathrm{R})$ irreps of the very different deformations, 


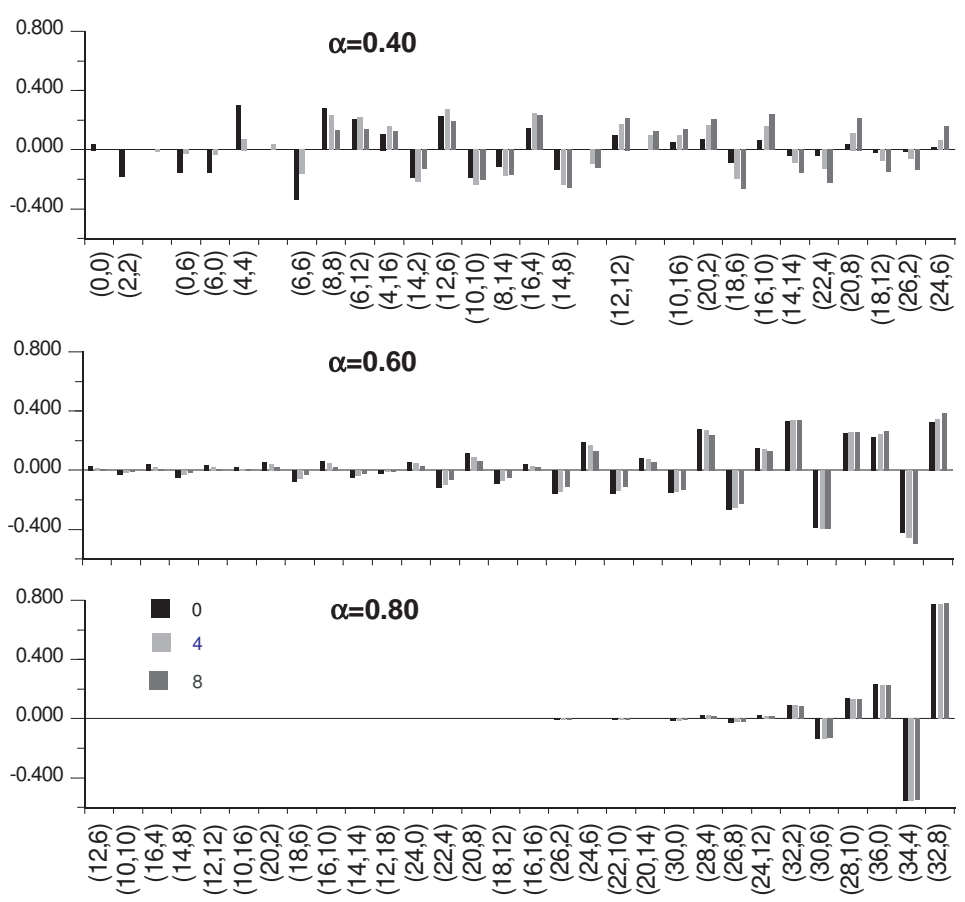

$(\lambda \mu)$

FIGURE 14. Histograms of the SU(3) amplitudes for the lowest energy $L=0$ to 8 eigenstates of the Hamiltonian (77) for four values of $\alpha$.

shown in Table 1, are precisely zero. These irreps can only mix indirectly via higherlying irreps. For example, the lowest-grade states of the excited irreps of ${ }^{12} \mathrm{C}$ and ${ }^{16} \mathrm{O}$ require the excitation of four particles relative to the ground-state irrep. Consequently, all matrix elements between states of the dominant irreps contributing to the ground and excited states are identically zero for a Hamiltonian with only two-body interactions.

\section{SUMMARY AND DISCUSSION}

I hope to have demonstrated in these talks that the well-developed group-theoretical and algebraic methods relating to symmetry provide important insights and powerful tools for investigating nuclear structure. In conjunction with geometry, analysis, and other standard tools, such as mean-field and coherent-state theory, they provide fundamental tools for studying the many interesting phenomena displayed in nuclear physics.

A message I want to emphasise is the importance of determining the domain of validity of a successful phenomenological model and of pursuing its relationship with other more sophisticated models, e.g., the many-nucleon theory of the nucleus. Investigating the limitations of a model is every bit as important as investigating its successes; it is probably more important. And establishing its microscopic foundations is what enables the model to contribute to the deeper understanding of the system it models.

I have focused on the emergence of the symplectic model, exposed its roots in the Bohr collective model, and pursued its role in the development of a microscopic shell- 
model theory of rotational nuclei. However, I would not want to create the impression that the symplectic model stands alone. The primary pillars of nuclear structure remain. They include: the shell model, the collective model, the unified models, the pairing model, and the Hartree-Fock and Hartree-Bogolyubov mean-field theories. The symplectic model builds on these foundations and, in parallel with the algebraic pairing model, it defines a shell-model coupling scheme that enables one to make use of what one learns, from model fits to data, in the design of a more complete microscopic theory. Like the pairing model, which supplies the standard $J J$ coupling scheme of the spherical shell model and provides a shell-model theory of singly-closed shell nuclei, the symplectic model supplies a coupling scheme that relates to shell-model bases expressed both in terms of spherical harmonic oscillator and anisotropic harmonic oscillator wave functions. Thus, as discussed in the text, it provides a shell-model theory of particular relevance to strongly deformed rotational states of nuclei.

Some possibly novel perspectives have been presented. However, what is satisfying that the symplectic model embraces the views assembled from the vantage points of many developments and sets them in a new light. For example, an extension of the spherical shell-model with spherical harmonic oscillator wave functions to the Nilsson model [11] with anisotropic harmonic oscillator wave functions was made already in 1955. However, the extension was designed only for the purpose of describing the states of added nucleons interacting with a deformed rotational core nucleus. Nevertheless, it was envisioned by many that the Nilsson model should somehow be extendable to a shell-model for deformed nuclei with non-spherical basis wave functions. The symplectic model, which relates many-particle Nilsson model states to lowest-weight states of $\operatorname{Sp}(3, \mathrm{R})$ irreps, makes this vision a reality. Moreover, as we have discussed, it leads to a practical generator coordinate approach to $\operatorname{Sp}(3, \mathrm{R})$ model calculations with microscopic interactions and approximations to mixed representation calculations with the inclusion of spin-orbit and other $\operatorname{Sp}(3, \mathrm{R})$ symmetry-breaking interactions.

The symplectic model gives the deformed shell model a fundamental foundation. In particular, it resolves concerns about completeness and orthogonality. In the first place, states associated with different $\mathrm{Sp}(3, \mathrm{R})$ irreps and different angular momenta and spin are strictly orthogonal. Moreover, it is known that a geometric space of $\operatorname{Sp}(3, \mathrm{R})$ coherent states spans the Hilbert space for an $\operatorname{Sp}(3, \mathrm{R})$ irrep. Thus, the states of each such space are orthogonal to all other such spaces and, together, they span the whole many-nucleon Hilbert space. Thus, it is only necessary to orthogonalise basis states with common values for the associated quantum numbers. This is an enormous advantage over similar projected Hartree-Fock methods both computationally and conceptually; e.g., it retains a knowledge of what shell model spaces and $\operatorname{Sp}(3, \mathrm{R})$ irreps are included in a calculation.

In conclusion, it should be emphasised that the construction of a shell-model theory of doubly open-shell nuclei is far from a minor topic in nuclear structure physics. Certainly doubly closed- and singly closed-shell nuclei have contributed a lot to the understanding of nuclear structure. However, such nuclei represent a small fraction of the nuclei available for study. Moreover, it is rapidly becoming apparent [22] that strongly deformed states, generally associated with doubly-open shell nuclei, are common among the excited states of singly and doubly closed-shell nuclei in which both the neutron and proton degrees become active, 


\section{REFERENCES}

1. B. H. Flowers, Proc. Roy. Soc. London A212, 248-263 (1952).

2. J. P. Elliott, Proc. Roy. Soc. (London) A245, 128, 562 (1958).

3. A. Bohm, Y. Ne'eman, and A. O. Barut, editors, Dynamical Groups and Spectrum Generating Algebras, vol. 1 and 2, World Scientific, Singapore, 1988.

4. D. J. Rowe, and P. S. Turner, Nucl. Phys. A753, 94-105 (2005).

5. M. A. Caprio, D. J. Rowe, and T. A. Welsh, Comp. Phys. Comm. 180, 1150-1163 (2009).

6. D. J. Rowe, T. A. Welsh, and M. A. Caprio, Phys. Rev. C 79, 054304(16) (2009).

7. T. A. Welsh, and D. J. Rowe, A computer code for calculations in the algebraic collective model of the atomic nucleus (in preparation) (2013).

8. D. Rowe, J. Phys. A: Math. Gen. 38, 10181-10201 (2005).

9. D. J. Rowe, P. S. Turner, and J. Repka, J. Math. Phys. 45, 2761-2784 (2004).

10. A. Bohr, and B. R. Mottelson, Nuclear Structure, vol. 2, Benjamin, Reading, Mass., 1975, (republished by World Scientific, Singapore).

11. S. G. Nilsson, Mat. Fys. Medd. Dan. Vid. Selsk. 29 (1955).

12. L. Weaver, L. C. Biedenharn, and R. Y. Cusson, Ann. Phys. (N.Y.) 77, 250-278 (1973).

13. D. J. Rowe, and G. Rosensteel, Ann. Phys. (N.Y.) 126, 198-233 (1980).

14. G. Rosensteel, and D. J. Rowe, Ann. Phys. (NY) 96, 1-42 (1976).

15. D. J. Rowe, and G. Rosensteel, J. Math. Phys. 20, 465-468 (1979).

16. G. Rosensteel, and D. J. Rowe, Phys. Rev. Lett. 38, 10-14 (1977).

17. G. Rosensteel, and D. J. Rowe, Ann. Phys. (NY) 126, 343-370 (1980).

18. G. Rosensteel, and D. J. Rowe, Phys. Rev. Lett. 47, 223-226 (1981).

19. R. Le Blanc, J. Carvalho, and D. J. Rowe, Phys. Lett. B 140, 155-158 (1984).

20. D. J. Rowe, M. G. Vassanji, and M. J. Carvalho, Nucl. Phys. A 504, 76-102 (1989).

21. M. J. Carvalho, R. Le Blanc, M. G. Vassanji, D. J. Rowe, and J. McGrory, Nucl. Phys. A 452, 240-262 (1986).

22. K. Heyde, and J. L. Wood, Rev. Mod. Phys. 83, 1467-1521 (2011).

23. C. Bahri, and D. J. Rowe, Nucl. Phys. A 662, 125-147 (2000).

24. M. Jarrio, J. L. Wood, and D. J. Rowe, Nucl. Phys. A 528, 409-435 (1991).

25. D. J. Rowe, Phys. Rev. 162, 866-871 (1967).

26. R. Le Blanc, J. Carvalho, M. G. Vassanji, and D. J. Rowe, Nucl. Phys. A 452, 263-276 (1986).

27. D. J. Rowe, G. Thiamova, and J. L. Wood, Phys. Rev. Lett. 97, 202501(4) (2006).

28. A. Dreyfuss, K. D. Launey, C. Bahri, T. Dytrych, and J. P. Draayer, "A Microscopic Description of the Elusive Hoyle State," in APS Division of Nuclear Physics Meeting Abstracts, 2011.

29. A. C. Dreyfuss, K. D. Launey, T. Dytrych, J. P. Draayer, and C. Bahri, submitted (2012).

30. D. J. Rowe, J. Math. Phys. 25, 2662-2671 (1984).

31. D. J. Rowe, G. Rosensteel, and R. Carr, J. Phys. A: Math. Gen. 17, L399-L403 (1984).

32. D. J. Rowe, G. Rosensteel, and R. Gilmore, J. Math. Phys. 26, 2787-2791 (1985).

33. D. J. Rowe, Rep. Prog. Phys. 48, 1419-1480 (1985).

34. R. D. Ratna Raju, J. P. Draayer, and K. T. Hecht, Nucl. Phys. A 202, 433-466 (1973).

35. D. Troltenier, C. Bahri, and J. Draayer, Nucl. Phys. A 586, 53-72 (1995).

36. T. Dytrych, K. D. Sviratcheva, C. Bahri, J. P. Draayer, and J. P. Vary, J. of Phys. G: Nucl. Part. Phys. 35, 095101(11) (2008).

37. T. Dytrych, K. D. Sviratcheva, J. P. Draayer, C. Bahri, and J. P. Vary, J. of Phys. G: Nucl. Part. Phys. 35, 123101(47) (2008).

38. G. F. Filippov, and I. P. Okhrimenko, Sov. J. Nucl. Phys. 32, 37 (1980).

39. V. S. Vasilevsky, Y. F. Smirnov, and G. F. Filippov, Yad. Fiz 32, 987-997 (1980), [English translation: Sov. J. Nucl. Phys. 32 510-515].

40. M. G. Vassanji, and D. J. Rowe, Phys. Lett. B 115, 77-80 (1982).

41. M. G. Vassanji, and D. Rowe, Nucl. Phys. A 426, 205-221 (1984).

42. M. J. Carvalho, M. G. Vassanji, and D. J. Rowe, Phys. Lett. B 318, 273-276 (1993).

43. M. J. Carvalho, and D. J. Rowe, Nucl. Phys. A 618, 65-86 (1997).

44. M. J. Carvalho, D. J. Rowe, S. Karram, and C. Bahri, Nucl. Phys. A 703, 167-187 (2002).

45. R. Y. Cusson, and H. C. Lee, Nucl. Phys. A pp. 429-462 (1973).

46. D. J. Rowe, "Compatible and incompatible symetries in the theory of nuclear collective motion," 
in New Perspectives in Nuclear Structure, edited by A. Covello, Fifth International Spring Seminar, Ravello, 1995, World Scientific, Singapore, 1996.

47. M. Born, and J. R. Oppenheimer, Ann. Physik. 84, 457- (1927), [translation by S. M. Binder in URL http://www.ulb.ac.be/cpm/people/scientists/bsutclif/bornop.pdf].

48. D. J. Rowe, P. Rochford, and J. Repka, J. Math. Phys. 29, 572-577 (1988).

49. D. J. Rowe, "Embedded Representations and Quasi-Dynamical Symmetry," in Computational and Group-Theoretical Methods in Nuclear Physics, edited by J. Escher, O. Castanos, J. Hirsch, S. Pittel, and G. Stoitcheva, World Scientific, Singapore, 2004, pp. 165-173, arXiv:1106.1607 [nucl-th].

50. P. Cejnar, J. Jolie, and R. F. Casten, Rev. Mod. Phys. 82, 2155-2209 (2010).

51. C. Bahri, D. J. Rowe, and W. Wijesundera, Phys. Rev. C 58, 1539-1550 (1998). 